\title{
Genetic Analysis of Histamine Signaling in Larval Zebrafish Sleep
}

\author{
[DAudrey Chen, ${ }^{1}{ }^{\circledR}$ Chanpreet Singh, ${ }^{1}$ Grigorios Oikonomou, ${ }^{1}$ and ${ }^{-D a v i d ~ A . ~ P r o b e r ~}{ }^{1}$ \\ DOI:http://dx.doi.org/10.1523/ENEURO.0286-16.2017 \\ ${ }^{1}$ Division of Biology and Biological Engineering, California Institute of Technology, Pasadena, CA 91125
}

\begin{abstract}
Pharmacological studies in mammals and zebrafish suggest that histamine plays an important role in promoting arousal. However, genetic studies using rodents with disrupted histamine synthesis or signaling have revealed only subtle or no sleep/wake phenotypes. Studies of histamine function in mammalian arousal are complicated by its production in cells of the immune system and its roles in humoral and cellular immunity, which can have profound effects on sleep/wake states. To avoid this potential confound, we used genetics to explore the role of histamine in regulating sleep in zebrafish, a diurnal vertebrate in which histamine production is restricted to neurons in the brain. Similar to rodent genetic studies, we found that zebrafish that lack histamine due to mutation of histidine decarboxylase ( $h d c)$ exhibit largely normal sleep/wake behaviors. Zebrafish containing predicted null mutations in several histamine receptors also lack robust sleep/wake phenotypes, although we are unable to verify that these mutants are completely nonfunctional. Consistent with some rodent studies, we found that arousal induced by overexpression of the neuropeptide hypocretin (Hcrt) or by stimulation of hcrt-expressing neurons is not blocked in $h d c$ or hrh1 mutants. We also found that the number of hcrt-expressing or histaminergic neurons is unaffected in animals that lack histamine or Hcrt signaling, respectively. Thus, while acute pharmacological manipulation of histamine signaling has been shown to have profound effects on zebrafish and mammalian sleep, our results suggest that chronic loss of histamine signaling due to genetic mutations has only subtle effects on sleep in zebrafish, similar to rodents.
\end{abstract}

Key words: genetics; histamine; hypocretin; sleep; wake

\section{Significance Statement}

Based on pharmacological studies in several model organisms, histamine is thought to be a key arousalpromoting neuromodulator. However, genetic studies in rodents have reported only subtle phenotypes. Rodent studies are complicated by involvement of histamine in regulating the immune system, which itself affects sleep. In zebrafish, histamine production is restricted to neurons in the brain, thus allowing study of histamine function in arousal without confounding effects of abnormal immune system function. We show that zebrafish lacking histamine synthesis have largely normal sleep/wake behaviors, as do histamine receptor mutants, although we lack tools to verify that the receptor mutants are nonfunctional. These results suggest that genetic loss of histamine signaling has little effect on sleep/wake behaviors in zebrafish, similar to rodents.

\section{Introduction}

Sleep is an essential and evolutionarily conserved behavioral state whose regulation remains poorly under-

Received September 22, 2016; accepted February 9, 2017; First published February 15, 2017

The authors declare no competing financial interests. stood. Histamine, which is thought to promote arousal, is synthesized by histidine decarboxylase ( $\mathrm{Hdc})$, which is expressed in the hypothalamic tuberomammillary nucleus 
(TMN) (Thakkar, 2011). In mammals, histamine activates four receptor subtypes (Seifert et al., 2013). Histamine H1 receptor (Hrh1) is expressed widely in the brain, particularly in regions implicated in neuroendocrine, behavioral, and nutritional state control (Panula et al., 2015). Hrh2 is also expressed in several brain regions, as well as in peripheral tissues where it regulates gastric acid secretion, heart function, and the immune system (Panula et al., 2015). Hrh3 can act both as an autoreceptor that inhibits the synthesis and release of histamine and as a heteroreceptor that inhibits the release of other neurotransmitters (Schlicker et al., 1994; Morisset et al., 2000; Arrang et al., 2007; Haas et al., 2008; Samaranayake et al., 2016). In humans, Hrh3 is strongly expressed in the basal ganglia, hippocampus, and cortical areas (Martinez-Mir et al., 1990). Hrh4 is exclusively expressed in the periphery, where it functions in mast cells, eosinophils and T cells to mediate inflammatory, itch, and immune responses (de Esch et al., 2005).

Several studies have suggested that histamine promotes wakefulness in mice and Drosophila. For example, animals treated with Hdc inhibitors or Hrh1 antagonists exhibit sedation (Roth et al., 1987; Thakkar, 2011). However, mutants with disrupted histamine synthesis or signaling exhibit remarkably normal sleep/wake behaviors, primarily exhibiting subtle defects in wakefulness when high vigilance is required (Inoue et al., 1996; Kubota et al., 2002; Parmentier et al., 2002; Abe et al., 2004; Anaclet et al., 2009; Thakkar, 2011). The basis of the discrepancy between genetic and pharmacological manipulations remains unclear, although similar discrepancies have been reported for other sleep regulatory systems. For example, drugs that affect signaling via adenosine or noradrenergic receptors have significant effects on sleep, whereas mutations that affect adenosine or noradrenergic signaling result in only subtle phenotypes, in rodents (Berridge et al., 2012; Brown et al., 2012). Studying the role of histamine in mammalian arousal is complicated because, in addition to its neurologic functions, it also plays a central role in humoral and cellular immunity (Haas et al., 2008; Jutel et al., 2009). The zebrafish has recently emerged as a useful model organism to study sleep (Chiu and Prober, 2013) and may be particularly appropriate to explore the role of histamine in arousal, because histamine production is restricted to the TMN (Da'as et al., 2011) and the zebrafish genome appears to lack an or-

This work was supported by National Institutes of Health Grants NS060996, NS070911, DA031367, and NS094390; the Mallinckrodt Foundation; the Rita Allen Foundation; and the Brain and Behavior Research Foundation.

A.C.'s present address: W.M. Keck Science Center of Claremont McKenna, Pitzer and Scripps Colleges, Claremont, CA 91711

Acknowledgements: We thank Daisy Chilin and Axel Dominguez for animal husbandry assistance and Laura Glass, Viveca Sapin, and Uyen Pham for technical assistance.

Correspondence should be addressed to David A. Prober at the above address, E-mail: dprober@caltech.edu.

DOI:http://dx.doi.org/10.1523/ENEURO.0286-16.2017

Copyright () 2017 Chen et al.

This is an open-access article distributed under the terms of the Creative Commons Attribution 4.0 International, which permits unrestricted use, distribution and reproduction in any medium provided that the original work is properly attributed. tholog of the peripherally expressed Hrh4 (Peitsaro et al., 2007). Furthermore, with only 10-12 histaminergic neurons, zebrafish larvae provide a simpler model to explore the role of these neurons in sleep (Sundvik et al., 2011). Previous studies showed that Hrh1 antagonists produce similar robust effects on zebrafish sleep as in mammals (Peitsaro et al., 2007; Renier et al., 2007; Rihel et al., 2010; Sundvik et al., 2011), but genetic manipulation of histamine signaling in zebrafish has not been reported.

To address the role of histamine in regulating sleep/ wake states in a vertebrate animal that lacks histamine in the immune system, we generated zebrafish containing predicted null mutations in hdc and in histamine receptors. Similar to rodent $h d c$ and $h r h$ mutants (Inoue et al., 1996; Yanai et al., 1998; Parmentier et al., 2002; Abe et al., 2004; Anaclet et al., 2009), we found that sleep/wake states are largely normal in each larval zebrafish mutant. In contrast to one rodent report (Huang et al., 2001), but consistent with others (Carter et al., 2009; Hondo et al., 2010), we found that histamine is not required for arousal induced by overexpression of the neuropeptide hypocretin (Hcrt) or by stimulation of hcrt-expressing neurons in zebrafish larvae. Finally, in contrast to previous morpholino and pharmacology studies (Sundvik et al., 2011), we found that genetic blockage of Hcrt or histamine signaling does not affect the number of histaminergic or hortexpressing neurons, respectively. Together with previously described effects of pharmacological manipulation of histamine signaling in zebrafish (Peitsaro et al., 2007; Renier et al., 2007; Rihel et al., 2010; Sundvik et al., 2011), our results suggest that the role of histamine in regulating sleep/wake states is largely similar in zebrafish and mammals.

\section{Materials and Methods}

\section{Zebrafish genetics}

All animal procedures followed standard protocols (Westerfield, 2000) in accordance with the California Institute of Technology Institutional Animal Care and Use Committee guidelines. For most experiments, heterozygous adults were crossed to obtain homozygous mutant, heterozygous mutant and wild-type (WT) sibling larvae, which were genotyped after imaging or behavioral experiments. In cases where two or more mutants and/or transgenic animals were compared, we mated heterozygous adults to homozygous adults to reduce the number of genotypes present in their progeny and, thus, increase the number of animals of each genotype in the experiment. This was necessary to achieve sufficient animals of each genotype to obtain statistically robust data. This comparison is reasonable because heterozygous mutant animals did not exhibit phenotypes compared with their WT siblings. All experiments were performed using zebrafish larvae before the onset of sexual differentiation. hdc (RRID: ZDB-GENE-080102-5) mutant: TALEN binding sites were 5'-TCACTGCTGGGAGACA-3' and 5'-TGAAGCCGAGGCAGTT-3'. hdc mutant d10 contains a 10-bp deletion (TGCTGGCAGA) after nucleotide 277 of the open reading frame. The mutation results in a change in reading frame after amino acid 92 and a premature stop codon after amino acid 164, compared with 608 amino acids 
for the WT protein. The predicted mutant protein lacks conserved residues that are required for function of the human hdc ortholog (Komori et al., 2012). hdc mutants were genotyped using the primers 5'-TACCCAGGTGAAGCCGAG-3' and 5'-GCTGCAGTTCTGCTGTGTGT-3', followed by digest with BsaHI (New England Biolabs), which cuts the 144-bp WT PCR product into 114 and 30 bp. hrh1 (RRID: ZDBGENE-070531-3) mutant: the hrh1 hu3427 mutant was generated by the Zebrafish Mutation Project (Kettleborough et al., 2013) and contains an ATT nonsense mutation at nucleotide 1366 of the open reading frame, which is predicted to generate a 456-amino acid protein compared with the 534-amino acid WT protein. The mutant protein lacks two transmembrane domains and should thus be nonfunctional. hrh1 mutants were genotyped using the primers 5'-TCCGCTGGACGCTAGTATTG-3' and 5'-AGCCCAGCTGGCGCGCCGCTTTCCTCTCTT-3', followed by digest with Ddel (New England Biolabs), which cuts the 125-bp mutant PCR product into 95 and 30 bp. hrh2a (RRID: ZDB-GENE-070531-4) mutant: TALEN binding sites were 5'-TCATCCTGCTCACTGTAA-3' and 5'TAGCATACACAGCCAGAC-3'. hrh2a mutant d10 contains a 10-bp deletion (AATATTCTGG) after nucleotide 63 of the open reading frame. The mutation results in a change in reading frame after amino acid 21 and a premature stop codon after amino acid 42, compared with 369 amino acids for the WT protein. The predicted mutant protein lacks six transmembrane domains and should thus be nonfunctional. hrh2a mutants were genotyped using the primers 5'CTITAGCTGTGACGCTCTCC-3' and 5'-GCTAGCGAAACGATGAAGCA-3', which produces a 124-bp PCR product for WT and a 114-bp product for the mutant. hrh2b (RRID: ZDB-GENE-070928-20) mutant: TALEN binding sites were 5'-TGACAGACCTACTTCT-3' and 5'-TCCAGCATGGCAGAAAGT-3'. hrh2b mutant d8 contains an 8-bp deletion (TTGCTAGT) after nucleotide 162 of the open reading frame. The mutation results in a change in reading frame after amino acid 54 and a premature stop codon after amino acid 96, compared with 335 amino acids for the WT protein. The predicted mutant protein lacks six transmembrane domains and should thus be nonfunctional. hrh $2 b$ mutants were genotyped using 5'-CTGGTTTGTATGGCCGTGG-3' and 5'TTTCCATTGCGCAGTTCCAG-3', which produces a 140-bp PCR product for WT and 132 bp for the mutant. hrh3 (RRID: ZDB-GENE-040724-204) mutant: ZFN binding sites were 5'-TCCGTGGCG-3' and 5'-GCAGTCCTC-3'. hrh3 mutant d4 contains a 4-bp deletion (GTGG) after nucleotide 1022 of the open reading frame. The mutation results in a change in reading frame after amino acid 341 and a premature stop codon after amino acid 372 , compared with 473 amino acids for the WT protein. The predicted mutant protein lacks two transmembrane domains and should thus be nonfunctional. hrh3 mutants were genotyped using the primers 5'GAAACGGTTGGCTAGACTGG-3', 5'-CTTGCCTCCTCTGCAGAA-3', and 5'-TGGCTTCAACCGCTAAAGTG-3', which generate one band for WT (206 bp), two bands for homozygous mutant (202 and $123 \mathrm{bp}$ ), and three bands for heterozygous mutant (206, 202, and $123 \mathrm{bp})$.

Sequence alignments were performed using MegAlign Pro (DNASTAR).
The hcrtr hu2098 mutant line (RRID: ZDB-ALT-07042714) was generated by the Zebrafish Mutation Project (Kettleborough et al., 2013) and has previously been described (Yokogawa et al., 2007). hcrtr mutants were genotyped using the primers 5'-CCACCCGCTAAAATTCAAAAGCACTGCTAAC-3' and 5'-CATCACAGACGGTGAACAGG-3', followed by digest with Ddel, which cuts the 170-bp mutant PCR product into 140 and $30 \mathrm{bp}$.

The Tg(hsp:Hcrt) line (RRID: ZDB-TGCONSTRCT070228-2) has previously been described (Prober et al., 2006). Transgenic animals were identified using the primers 5'-CGGGACCACCATGGACT-3' and 5'-GGTTTGTCCAAACTCATCAATGT-3', which generate a 470-bp PCR product.

Tg(hcrt:ReaChR-mCitrine): the 1-kb zebrafish hort promoter (Faraco et al., 2006) was amplified using the primers 5'-ATAATAAATAAATCTGATGGGGTTTT-3' and 5'GAGTTTAGCTTCTGTCCCCTG-3', and subcloned 5' to a transgene encoding the channelrhodopsin-2 variant ReaChR (Lin et al., 2013) fused to mCitrine, in a plasmid containing flanking Tol2 transposase sequences, using Gibson assembly. Stable transgenic lines were generated using the Tol2 transposase method (Asakawa and Kawakami, 2009). Transgenic animals were identified using fluorescence or by PCR using the primers: 5'CACGAGAGAATGCTGTTCCA-3' and 5'-CCATGGTGCGTTTGCTATAA-3', which generate a 431-bp product.

\section{Histamine ELISA}

Adult fish were anesthetized in $0.2 \%$ tricaine and euthanized in ice water for 15 min before dissection in chilled PBS. Triplicate samples containing five adult brains each were homogenized in $160 \mu \mathrm{L}$ of $0.2 \mathrm{~N}$ perchloric acid. Homogenate was spun at $4^{\circ} \mathrm{C}$ at $10 \times 10^{3} \mathrm{~g}$ for $5 \mathrm{~min}$ and applied to a $0.45-\mu \mathrm{m}$ filter (UFC30HV00; Millipore). Filtrate was centrifuged at $4^{\circ} \mathrm{C}$ at $10 \times 10^{3} \mathrm{~g}$ for $15 \mathrm{~min}$. A total of $150 \mu \mathrm{L}$ of the supernatant was collected and neutralized by adding $150-\mu \mathrm{L} 1 \mathrm{M} \mathrm{K}_{2} \mathrm{~B}_{4} \mathrm{O}_{7}$, pH 8.0. Histamine levels were assayed using a histamine ELISA kit (IM2015; Immunotech) according to the manufacturer's instructions. Absorbance was read at $405 \mathrm{~nm}$ using a plate reader (Infinite M200Pro; Tecan). A calibration curve was generated by fitting absorbance values of manufacturerprovided standards to a 4-parameter logistic curve. In some cases, histamine readings of $h d c-/-$ samples were lower than the $0 \mathrm{mM}$ standard and were rounded up to 0 $\mathrm{mM}$.

\section{Immunohistochemistry (IHC)}

Five days postfertilization (dpf) larvae were fixed with freshly made, chilled $4 \% \mathrm{~N}$-Ethyl-N'-(3-dimethylaminopropyl) carbodiimide hydrochloride (EDAC), $0.1 \%$ paraformaldehyde (PFA) in $0.1 \mathrm{M}$ PBS for $16 \mathrm{~h}$ at $4^{\circ} \mathrm{C}$ and then washed $3 \times 5$ min in $0.25 \%$ Triton X-100 in PBS (PBTx). Brains were manually dissected and blocked for $2 \mathrm{~h}$ at room temperature in $1 \%$ normal goat serum $/ 1 \%$ DMSO/ PBTx. For histamine $\mathrm{IHC}$, brains were incubated in 1:10,000 rabbit antihistamine (AB5885, Millipore) in blocking solution overnight for three nights $(\sim 70 \mathrm{~h})$ at $4^{\circ} \mathrm{C}$ with gentle agitation. Brains were washed $10 \times 15$ min with $1 \%$ DMSO/PBTx and incubated in 1:500 goat anti-rabbit 
Alexa Fluor 488 in blocking solution overnight at $4^{\circ} \mathrm{C}$. Samples were washed $10 \times 15 \mathrm{~min}$ in $1 \%$ DMSO/PBTx, once in PBS and equilibrated in 50\% glycerol/PBS. Samples were mounted in 50\% glycerol/PBS. Images were acquired using an LD LCI Plan-Apochromat 25x Imm Corr DIC objective (0.8 NA; Zeiss) and a Zeiss 780 confocal microscope. Larval progeny of an $+/-$ incross were used, and animals were genotyped by PCR after imaging. To verify specific ReaChR-mCitrine expression in Tg(hcrt: ReaChR-mCitrine) larvae, IHC and imaging were performed as described above using rabbit anti-orexin $A$ (AB3704, 1:500; Millipore) and chicken anti-GFP (GFP1020, 1:400; AvesLabs). Alexa Fluor secondary antibodies were used (1:500 Alexa Fluor 568 anti-rabbit and 1:600 Alexa Fluor 488 anti-chicken, Life Technologies).

\section{In situ hybridization (ISH)}

Samples were fixed in 4\% PFA/PBS for $16 \mathrm{~h}$ at room temperature. ISH was performed using digoxygenin (DIG)labeled antisense riboprobes (DIG RNA Labeling kit; Roche) as previously described (Thisse and Thisse, 2008). Probes specific for hcrt (hcrt; Prober et al., 2006) and hdc (hdc; Eriksson et al., 1998) have been described. Images were acquired using a Zeiss Axiolmager M1 microscope. Larval progeny of an $+/-$ incross were used, and animals were genotyped by PCR after imaging.

\section{Sleep-wake analysis}

Mammalian sleep is typically monitored using electrophysiology, but this can be difficult to perform in nonmammalian systems. In these cases, behavioral criteria can be used to define sleep states (Campbell and Tobler, 1984; Allada and Siegel, 2008). First, sleep is usually observed as locomotor quiescence that occurs during specific periods of the circadian cycle. Second, animals exhibit an increased arousal threshold during sleep, which distinguishes sleep from quiet wakefulness. Third, sleep is rapidly reversible, and sleeping animals can be aroused by strong stimuli, thus distinguishing sleep from paralysis or coma. Fourth, sleep is controlled by a homeostatic system, which can be demonstrated by an increased need for sleep following sleep deprivation. Using these criteria, we and others (Prober et al., 2006; Elbaz et al., 2012) have shown that 1 or minute of inactivity corresponds to a sleep state in zebrafish larvae. Waking activity is defined as the amount of locomotor activity not including sleep periods. Sleep latency is defined as the amount of time between lights on or off and the first sleep bout.

Larvae were raised on a 14/10 h light/dark cycle at $28.5^{\circ} \mathrm{C}$ with lights on at 9 A.M. and off at 11 P.M. Individual larvae were placed into each well of a 96-well plate (7701-1651; Whatman) containing E3 embryo medium (5 $\mathrm{mM} \mathrm{NaCl}, 0.17 \mathrm{mM} \mathrm{KCl}, 0.33 \mathrm{mM} \mathrm{CaCl}_{2}$, and $0.33 \mathrm{mM}$ $\left.\mathrm{MgSO}_{4}, \mathrm{pH} 7.4\right)$ at $4 \mathrm{dpf}$ in the evening. Plates were sealed with an optical adhesive film (4311971; Applied Biosystems) to prevent evaporation. We have not observed adverse effects of sealing the plate on animal health or behavior, and rather we observe more robust behaviors that last longer in sealed plates. In nonsealed plates, there is significant evaporation of water from each well each day, and thus, changes in ion concentrations in the water. Thus, although we add fresh water to each well every day in nonsealed plates, there are still daily fluctuations in ion concentrations that likely lead to less robust health and behavior. The sealing process introduces air bubbles in some wells, which are discarded from analysis. Locomotor activity was monitored using a videotracking system with a Dinion one-third inch Monochrome camera (Dragonfly 2; Point Gray) fitted with a variable-focus megapixel lens (M5018-MP; Computar) and infrared filter. The movement of each larva was captured at $15 \mathrm{~Hz}$ and recorded using the quantization mode with 1-min integration time bins. The 96-well plate and camera were housed inside a custom-built Zebrabox (Viewpoint Life Sciences) that was continuously illuminated with infrared lights. The 96-well plate was housed in a chamber filled with recirculating water to maintain a constant temperature of $28.5^{\circ} \mathrm{C}$. The parameters used for movement detection were as follows: detection threshold, 15; burst, 29; freeze, 3 . These values are used by the software to detect and score movements. They do not have standard units and must be set empirically. Data were analyzed using custom Perl and Matlab (Mathworks) scripts, and statistical tests were performed using Prism (GraphPad) and Matlab.

To assay behavioral responses to an environmental challenge, we exposed $h d c$ and $h r h 1$ mutants to alternating periods of light and darkness. Larvae were transferred to a 96-well plate at $5 \mathrm{dpf}$ in the afternoon and were exposed to alternating 1-h periods of light and darkness for $24 \mathrm{~h}$ starting at 5 P.M. We compared the brief increases in locomotor activity that are induced by light onset and offset (Prober et al., 2006; Burgess and Granato, 2007) and also quantified total locomotor activity during each light and dark period, with data collected in 5 -s bins. Because light offset induces an increase in locomotor activity that lasts $\sim 10 \mathrm{~min}$, we excluded these data from dark period locomotor activity quantification. For the analysis presented in the paper, we quantified locomotor activity during light and dark periods averaged for five light/dark cycles at night because the behavioral response to light onset is most apparent at night, and because it is difficult to compare features of behavioral traces that encompass longer time periods. In addition, locomotor activity levels are higher for all genotypes during the day than at night. As a result, combining data from the day and night risked obscuring subtle mutant phenotypes, although data for light and dark periods averaged over the entire 24-h experiment yielded results similar to those shown in Figure 6 (data not shown).

For Hcrt overexpression experiments, videotracker analysis was initiated at $4 \mathrm{dpf}$. During the afternoon of 6 $\mathrm{dpf}$, the 96 -well plate was transferred to a $37^{\circ} \mathrm{C}$ water bath for $1 \mathrm{~h}$ to induce Hcrt overexpression. Preheat shock behavior was calculated from day 5 and night 5 , and postheat shock behavior was calculated from night 6 and day 7. Two-way ANOVA was used to examine effects of heat shock (pre vs post) and genotype on behavior. 


\section{Mechano-acoustic stimulus assay}

This assay was performed, and data were analyzed as described previously (Singh et al., 2015). The videotracking system was modified by adding an Arduino based automated driver to control two solenoids (28P-I-12; Guardian Electric) that delivered a tap to a 96-well plate containing larvae. This setup allowed us to drive the solenoids with voltage ranging from 0 to $20 \mathrm{~V}$ over a range of 4095 settings (from 0.01 to 40.95). We used taps ranging from a power setting of 1-36.31. Taps of 14 different intensities were applied in a random order from 12:30 to 7:30 A.M. during the fifth day or night of development with an intertrial-interval of $1 \mathrm{~min}$. Previous studies showed that a 15-s interval between repetitive stimuli is sufficient to prevent behavioral habituation (Burgess and Granato, 2007; Woods et al., 2014). The background probability of movement was calculated by identifying for each genotype the fraction of larvae that moved $5 \mathrm{~s}$ before all stimuli delivered during an experiment (14 different tap powers $\times 30$ trials per experiment $=420$ data points per larva; average background movement). This value was subtracted from the average response fraction value for each tap event (corrected response $=$ average response - average background movement). Tapping experiments with a 5-min intertrial interval were performed from 12:30 to 9:00 A.M. using a single tap intensity (3.02) to assess the response of sleeping animals to the stimulus. The response of larvae to the stimuli was monitored using the videotracking software and was analyzed using Matlab (The Mathworks) and Excel (Microsoft). Curve fitting was performed using the Variable Slope log(dose) response curve-fitting module of Prism (GraphPad).

\section{Optogenetic assay}

This assay was performed, and data were analyzed as described (Singh et al., 2015). The videotracking system was modified to include an array of three sets of blue LEDs (470 nm, MR-B0040-10S, Luxeon V-star) mounted $15 \mathrm{~cm}$ above and $7 \mathrm{~cm}$ away from the center of the 96-well plate to ensure uniform illumination. The LEDs were controlled using a custom built driver and software written in BASIC stamp editor. A power meter (1098293, Laser-check) was used before each experiment to verify uniform light intensity $(\sim 450 \mu \mathrm{W}$ at the surface of the 96-well plate). During the afternoon of the fifth day of development, single larvae were placed into each well of a 96-well plate as described above and placed in the videotracker in the dark for $8 \mathrm{~h}$. Larvae were then exposed to blue light for $30 \mathrm{~min}$, starting at 1 A.M. Three trials were performed during the night, with an intertrial interval of 3 h. Total activity for each larva was monitored for $30 \mathrm{~min}$ before and after light onset, with data collected in 10-s bins. Light onset caused a short burst of locomotor activity lasting for $\sim 30 \mathrm{~s}$ for all genotypes, so data obtained during the minute before and after light onset were excluded from analysis. A large burst of locomotor activity was also observed for all genotypes when the lights were turned off after the 30-min illumination period. These data were excluded from analysis and are not shown in the figures. Data from three independent experiments were merged before analysis. The total amount of locomotor activity of each larva during the 30 min of light exposure, excluding the minute after light onset, was divided by the average baseline locomotor activity for all larvae of the same genotype. The baseline period was defined as 30 min before light onset, excluding the minute before light onset.

\section{Statistics}

Data were analyzed using one-way ANOVA, two-way ANOVA, or $t$ tests, as appropriate. Tukey's HSD test was used in post hoc analyses that compared three or more genotypes. For mechano-acoustic experiments with 1-min intertrial intervals, statistical significance was assessed using the extra sum-of-squares $F$ test. For mechano-acoustic experiments with 5-min intertrial intervals, statistical significance was assessed using one-way ANOVA.

\section{Results}

\section{Generation of a zebrafish hdc mutant that lacks histamine}

Within the vertebrate central nervous system, histamine is produced exclusively in the TMN due to expression of hdc, which converts histidine to histamine (Schwartz et al., 1991). The zebrafish genome contains a single $h d c$ ortholog, which encodes for a 595-amino acid protein (Fig. 1A). Zebrafish larvae have 10-15 hdc-expressing neurons per brain hemisphere (Sundvik and Panula, 2015), compared with $\sim 5000$ in rodents (Ericson et al., 1987) and $\sim 60,000$ in humans (Airaksinen et al., 1991). To explore the role of histamine in regulating sleep in zebrafish, we generated a hdc mutant using the TALEN method (Sander et al., 2011). We isolated an hdc mutant containing a 10-bp deletion, which results in a predicted 164-amino acid protein that lacks conserved residues required for function of the human hdc ortholog (Komori et al., 2012; Fig. 1A,B). To determine whether zebrafish hdc mutants produce histamine, we performed IHC using a histamine-specific antibody. At $5 \mathrm{dpf}$, we observed robust antibody labeling in $14.9 \pm 0.8$ cells $(n=10$ larvae) in the TMN of $h d c+/+$ larvae (Fig. 1C). We observed a similar number of neurons in hdc+/- larvae $(13.8 \pm 1.3$ cells, $n=13$ larvae), although histamine levels were reduced compared with their hdc $+/+$ siblings (Fig. 1C). In contrast, histamine labeling was absent in hdc-/- larvae ( 0 cells, $n=6$ larvae) (Fig. 1C). Using ISH, we observed that the level of hdc mRNA and the number of hdcexpressing neurons were similar for $h d c+/+(19.6 \pm 0.7$ cells, $n=17)$ and $h d c+/-(17.7 \pm 0.4$ cells, $n=26)$ larvae (Fig. 1D). In $h d c-/-$ larvae, $h d c$ expression was slightly weaker and there were slightly fewer $h d c$-expressing cells (15.8 \pm 0.9 cells, $n=17, p<0.001$ compared with $h d c+/+$ by Tukey's HSD test), presumably due to nonsense-mediated mRNA decay (Isken and Maquat, 2007). These observations indicate that most TMN neurons are present in $h d c-/-$ larvae, but they do not produce histamine. We also measured histamine levels by ELISA in adults, and found that $h d c+/+$ animals have 214 $\pm 12 \mu \mathrm{g}$ histamine per brain ( $n=15$ animals), while little 


\section{A}

HDC_Hs HDC_Mm HDC Dr

HDC_Dr d10

HDC_Hs $\mathrm{HDC} \mathrm{Mm}$ HDC_Dr HDC_Dr d
MMEPEEYRE-

MMEPCEYREYREYYRA $M Q P Q E Y M L$ -

RGREMVDYICQYLSTVRERRVTPDVQPGYLRAQLPESAPEDPDSWDSIFGDIERIIMPGVVHWQ RGKEMVEY HQYLTG I ERRVVPDVQPGFMRPL - RGKEMVEYIHQYLTGIRERRVVPDVQPGFMRPLLPSSAPYEPEDWSTIMQDVEN I IMPGVVHWQ

HDC_HS TLIALLAARKNKILEMK- - TSEPDADESCLNARLVAYASDQAHSSVEKAGLISLVKMKFLPVDDNFSLRGEALQKAIEED HDC_Mm TLIALLAARKNKILAMK- - ACEPDANESSLNARLVAYTSDQAHSSVEKAGLISLVKIRFLPVDDNFSLRGEALQKAIEED HDC_Dr TLVALLAARKDRILQMKSEATHTDTDESVLNSRLVAYASDQAHSSVEKAGLISLVKIRFLQTDAVFSLRGETLQRAVEED

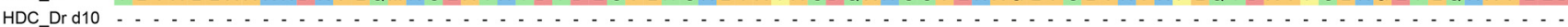

HDC_Hs KQRGLVPVFVCATLGTTGVCAFDCLSELGPICAREGLWLHIDAAYAGTAFLCPEFRGFLKGIEYADSFTFNPSKWMMVHF HDC $\mathrm{Mm}$ HDC_Dr HDC_Dr d10 KQQGLVPVFVCATLGTTGVCAFDRLSELGPICASEGLWLHVDAAYAGTAFLCPELRGFLEGIEYADSFTFNPSKWMMVHF RRSGLI PVMVCATLGSTGVCSFDRLDELGPVCVREGLWLHVDAAYAGSALLCPELRYFLDGIQFADSFVFNPSKWMLVHF

HDC_Hs HDC_Mm HDC_Dr HDC_Drd10

DCTGFWVKDKYKLQQTFSVNPIVLRHANSGVATDFMHWQIPLSRRFRSVKLWFVIRSFGVKNLQAHVRHGTEMAKYFESL DCTGFWVKDKYKLQQTFSVNPIYLRHAN SGAATDFMHWQI PLSRRFRSIKLWFVIRSFGVKNLQAHVRHGTEMAKYFESL DCTAFWVKNKMKLQQTFTVDPLYLRHDNSN- ATDFMHWQI PLSRRFRSLKLWFVIRSFGLKKLQEHIRHGVEMAKLFESL

HDC_Hs HDC_Mm HDC_Dr HDC_Dr d10

VRNDPSFEI PAKRHLGLVVFRLKGPNCLTENVLKEI AKAGRLFLIPATIQDKLI IRFTVTSQFTTRDDILRDWNLIRDAA VRSDPSFEI PAKRHLGLVVFRLKGPNCLTESVLKEIAKAGQLFLIPAT I QDKLIIRFTVTSQFT TKEDILRDWHLIQEAA VRNDTHFQ। PAQRHLGLVVFCLRAGNAATQELLRKLTRSGRMFLIPAAVGNQLILRFSVTSQLTTEQDIRRDWSLIQQAA

HDC Hs TLILSQHCTSQPSPRVGN I SQIRGARAWACGTSLQSVSGAGDDPVQARKIIKQPQRVGAGPMKRENGLHLETLLDPVDD HDC_Mm NLVLSQHCTSQPSPRAKNVIPPPPGTR - . - GLSLESVSEGGDDPAQARKIIKQP. - GASLARREGGSDLETMPDPFDD

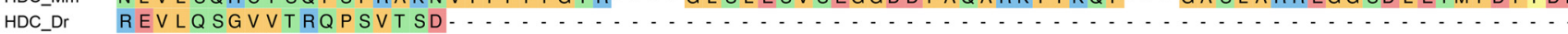

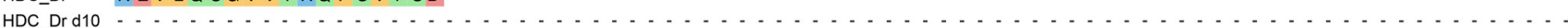

HDC_HS CFSEEAPDATKHKLSSFLFSYLSVQTKKKTVRSLSCNSVPVSAQKPLPTEASVKNGGSSRVRIFSRFPEDMMMLKKSAFK HDC Mm CFSEEAPNTTKHKLSSFLFSYLSVQNRRKTTRSLSCN SVPMSAQKSLPADASLKNGGSFRARIFSGFPEQMMMMKKGAFK HDC_Dr - P PESEPSLTIHTEPMLDQRLVRQGQRRAVRSYSCSA . . . ELPPAPGRTQTHRDAPLQLIPEQPE. - . - RPQHR

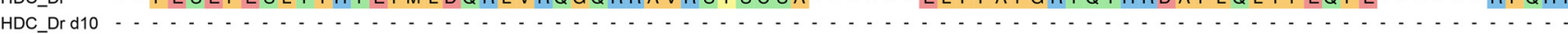

HDC_Hs KLIKFYSVPSFPECSSQCGLQ ....................

HDC_Mm KLIKFYSVPSFPECSSQCARQ.................. L LCCPLEAMV....

HDC_Dr RLLKFNSVPSLAQVWAQCGMQQLHHPFRRGLITSRASCFTCPPLPEGTPLPTK

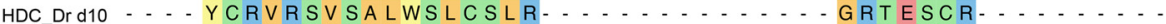

B

B

28.0

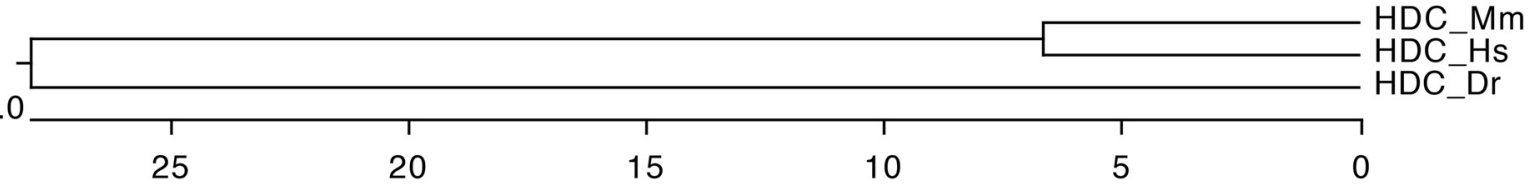

25

20
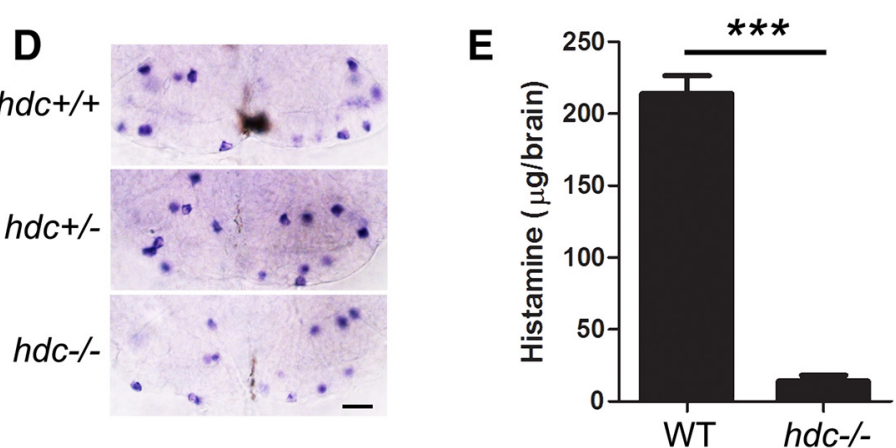

Figure 1. Characterization of hdc-/- zebrafish. A, Alignment of Hdc protein sequences from human (Hs, ENSG00000140287), mouse (Mm, ENSMUSG00000027360), zebrafish WT (Dr, ENSDARG00000075454), and zebrafish 10-bp deletion mutant (Dr d10). The red box indicates an amino acid required for the activity of human Hdc (Komori et al., 2012a). Amino acids are colored to indicate residues with similar properties. $\boldsymbol{B}$, Phylogenetic tree of human, mouse, and zebrafish Hdc. Values indicate the number of amino acid substitutions per 100 residues. $\boldsymbol{C}, \boldsymbol{D}$, Ventral views of 5 dpf larval brains in which histaminergic neurons are labeled using IHC with 
continued

a histamine-specific antibody $(\boldsymbol{C})$ or ISH using an $h d c$-specific riboprobe $(\boldsymbol{D})$ are shown for $h d c+/+, h d c+/-$, and $h d c-/-$ siblings. Histamine is reduced in $h d c+/-$ larvae and undetectable in $h d c-/-$ larvae $(\boldsymbol{C})$, but the number of hdc-expressing neurons is only slightly reduced in hdc-/- larvae compared with their hdc $+/+$ and $h d c+/-$ siblings $(\boldsymbol{D})$. Boxed region in schematic diagram (lower left) indicates region shown in $(\boldsymbol{C}, \boldsymbol{D})$, with the TMN shaded magenta. Scale bars: $20 \mu \mathrm{m}$. $\boldsymbol{E}$, Histamine concentration assayed by ELISA from WT and $h d c-/-$ adult brains. Little or no histamine was detected in hdc-/- fish. Histamine detected in hdc-/- animals is below the level of assay sensitivity and precision. $* * * p<0.001$ by Student's $t$ test.

or no histamine was detected in their hdc-/- siblings (Fig. 1E). These results indicate that larval and adult hdc-/- zebrafish produce little or no histamine.

\section{hdc mutant zebrafish larvae exhibit largely normal sleep/wake behaviors}

To determine whether histamine is required for normal larval zebrafish sleep/wake behaviors, we analyzed locomotor activity and sleep using a high-throughput behavioral assay (Prober et al., 2006). We observed that hdc-l- larvae exhibit similar levels of locomotor activity (Fig. $2 A, C, I)$ and waking activity (Fig. $2 D, J$ ) as their $h d c+/-$ and $h d c+/+$ siblings. Larvae of all three genotypes also exhibited similar total sleep amount during the day (Fig. 2B,E), as well as a similar number of sleep bouts (Fig. $2 F, L$ ) and sleep latency (Fig. $2 H, N$ ) during the day and night. $h d c+/-$ animals exhibited slightly decreased sleep and sleep bout length at night compared with their $h d c+/+$ siblings (Fig. $2 B, K, M$ ), the latter of which is consistent with the fragmented sleep reported for hdc-/- mice (Parmentier et al., 2002). The significance of these effects is unclear because these measures were not significantly different for hdc-/zebrafish compared with their $h d c+/+$ siblings. However, this discrepancy could be explained if compensatory mechanisms in $h d c-/-$ animals rescue effects of reduced histamine levels that are observed in $h d c+/-$ siblings (see discussion). Taken together, we conclude that zebrafish hdc mutants have largely normal total amounts of sleep and wakefulness in a 24-h period, similar to hdc knock-out mice (Parmentier et al., 2002). However, zebrafish hdc-/- larvae do not display decreased spontaneous activity or decreased sleep latency, as reported in some hdc knock-out mouse studies (Kubota et al., 2002; Parmentier et al., 2002; Abe et al., 2004; Anaclet et al., 2009).

To test whether the absence of behavioral phenotypes in $h d c-/-$ larvae is due to rescue by maternally deposited WT hdc mRNA, we mated hdc-/- females to $h d c+/-$ males and examined the behavior of their $h d c+/-$ and $h d c-/-$ progeny. We again observed no phenotypes in $h d c-/-$ larvae compared with their $h d c+/-$ siblings (Fig. $3 A-G, I-N$ ), except that $h d c-/-$ larvae had an increased latency to the first sleep episode following lights on in the morning (Fig. $3 H$ ), although this effect barely reached statistical significance. These results indicate that maternally contributed WT hdc mRNA may contribute modestly to daytime sleep latency, but otherwise does not account for the absence of sleep/wake phenotypes in zygotic hdc-l- larvae.

\section{hrh1 mutant larvae do not show major changes in sleep/wake behaviors}

While the hdc mutant data suggest that histamine is not required for normal sleep/wake behaviors in zebrafish larvae, histamine receptors exhibit constitutive ligand-independent signaling (Seifert et al., 2013), so we decided to explore potential roles for histamine receptors in larval zebrafish sleep. In mammals, histamine $\mathrm{H} 1$ receptor (Hrh1) is thought to mediate the effects of histamine on arousal because Hrh1 antagonists are sedating (Roth et al., 1987) and pretreatment with the Hrh1 antagonist pyrilamine blocks the arousing effect of histamine (Thakkar, 2011). hrh1 knock-out mice, however, exhibit reduced locomotor activity only when challenged with a novel environment (Inoue et al., 1996; Yanai et al., 1998) and surprisingly show increased activity during the day (Inoue et al., 1996), but otherwise exhibit largely normal sleep/wake behaviors (Huang et al., 2006).

The zebrafish genome appears to contain a single Hrh1 ortholog that is $40 \%$ identical to human Hrh1 (Fig. 4; Peitsaro et al., 2007). Similar to mammals, hrh1 is expressed in the ventral telencephalon, diencephalic and thalamic regions, and lateral hypothalamus in larval zebrafish (Sundvik et al., 2011). To determine whether Hrh1 is required for normal zebrafish sleep/wake states, we tested an hrh 1 mutant that contains a nonsense mutation and is predicted to generate a truncated protein that lacks two transmembrane domains, and should thus be nonfunctional (Fig. 4). $h r h 1-/-$ and $h r h 1+/-$ larvae exhibited slightly higher waking activity compared with their $h r h 1+/+$ siblings at night (Fig. 5J), similar to hrh1-/mice, which are hyperactive during the day, the rest period of these nocturnal animals (Inoue et al., 1996). Hrh1+/- larvae also showed increased waking activity during the day (Fig. 5D) and increased activity at night (Fig. 5/) compared with their $h r h 1+/+$ siblings, although the significance of these effects is unclear because they were absent in hrh1-/- larvae. We conclude that hrh1-/larvae lack major changes in sleep/wake behaviors compared with sibling controls, similar to $h r h 1-/-$ mice (Huang et al., 2006) and hdc-/- zebrafish (Figs. 2, 3).

\section{hdc and hrh1 mutant larvae exhibit normal behavioral responses to environmental challenges}

To explore whether environmental challenges uncover sleep/wake defects in hdc and hrh1 mutant zebrafish, as they do in rodents (Inoue et al., 1996; Yanai et al., 1998; 

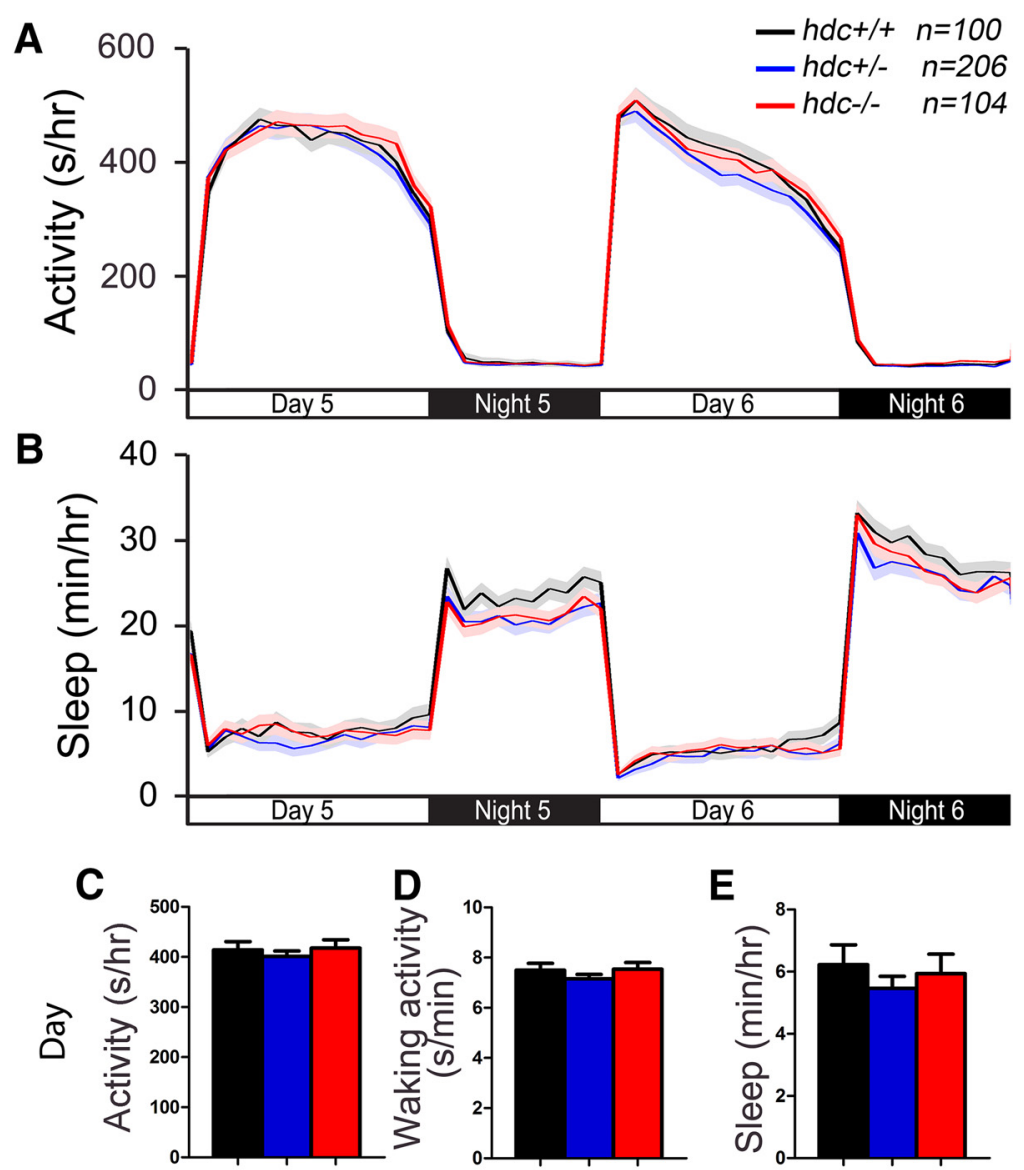

E
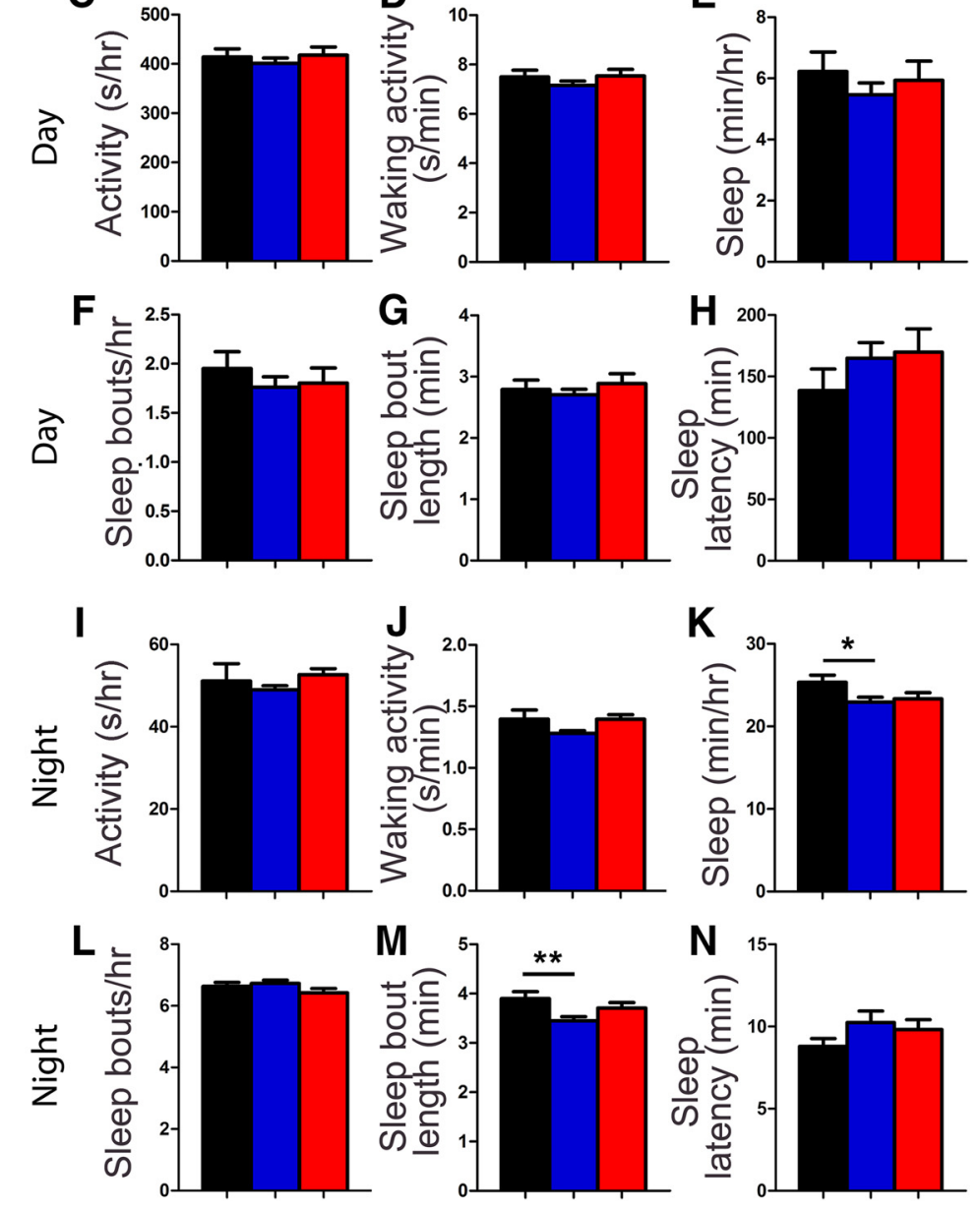

Figure 2. hdc mutant larvae exhibit normal sleep/wake behaviors. $h d c-/-($ red), $h d c+/-$ (blue) and $h d c+/+$ (black) siblings exhibit similar amounts of all measured parameters, except that $h d c+/-$ larvae sleep slightly less $(\boldsymbol{K})$ and have slightly shorter sleep bouts $(\boldsymbol{M})$ at night compared with their $h d c+/+$ siblings. Line and bar graphs represent the mean \pm SEM for five experiments combined; $n$ indicates the number of animals analyzed. $* p<0.05 ; * * p<0.01$ for the indicated comparisons by one-way ANOVA with Tukey's HSD test. 

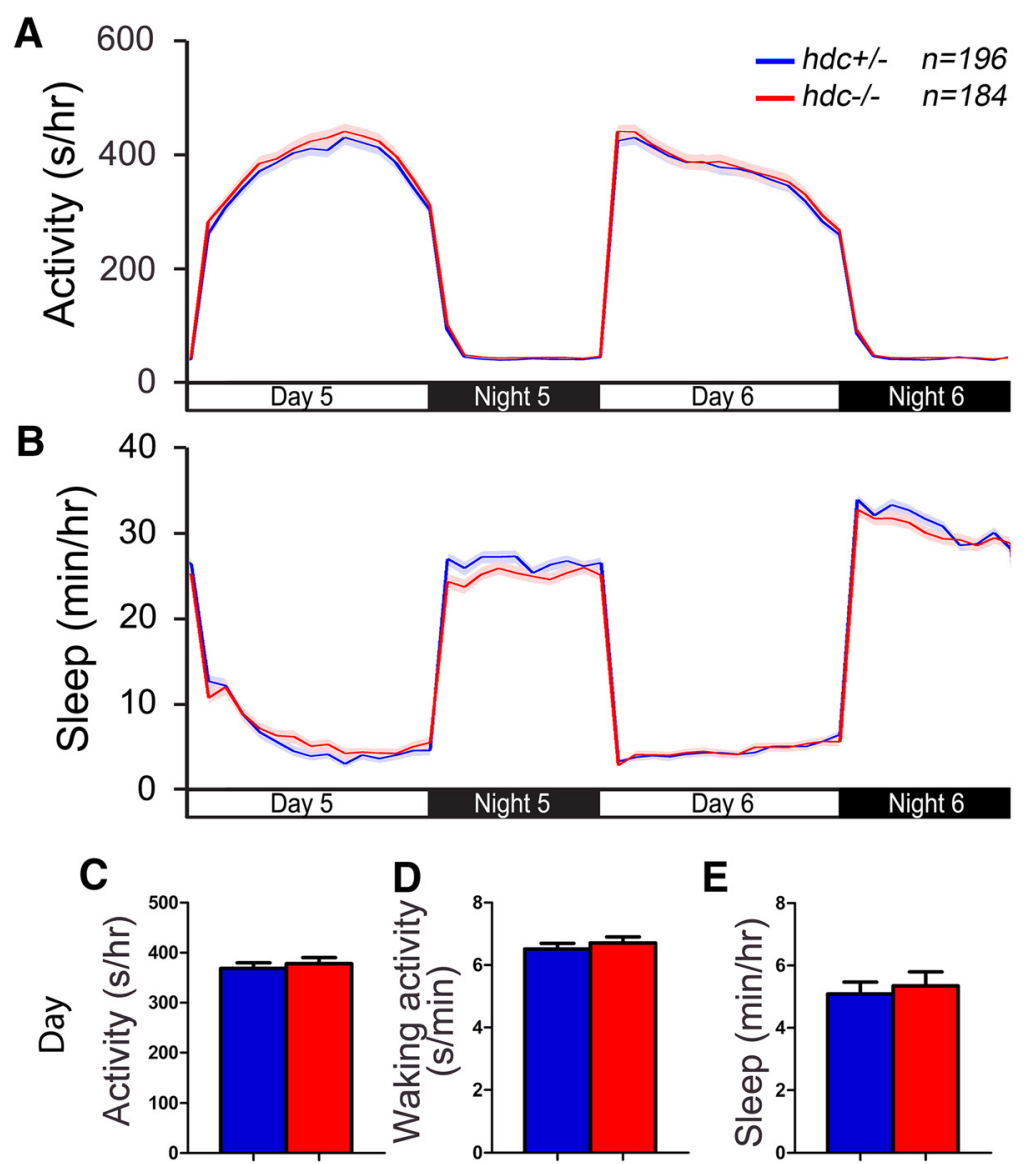

E
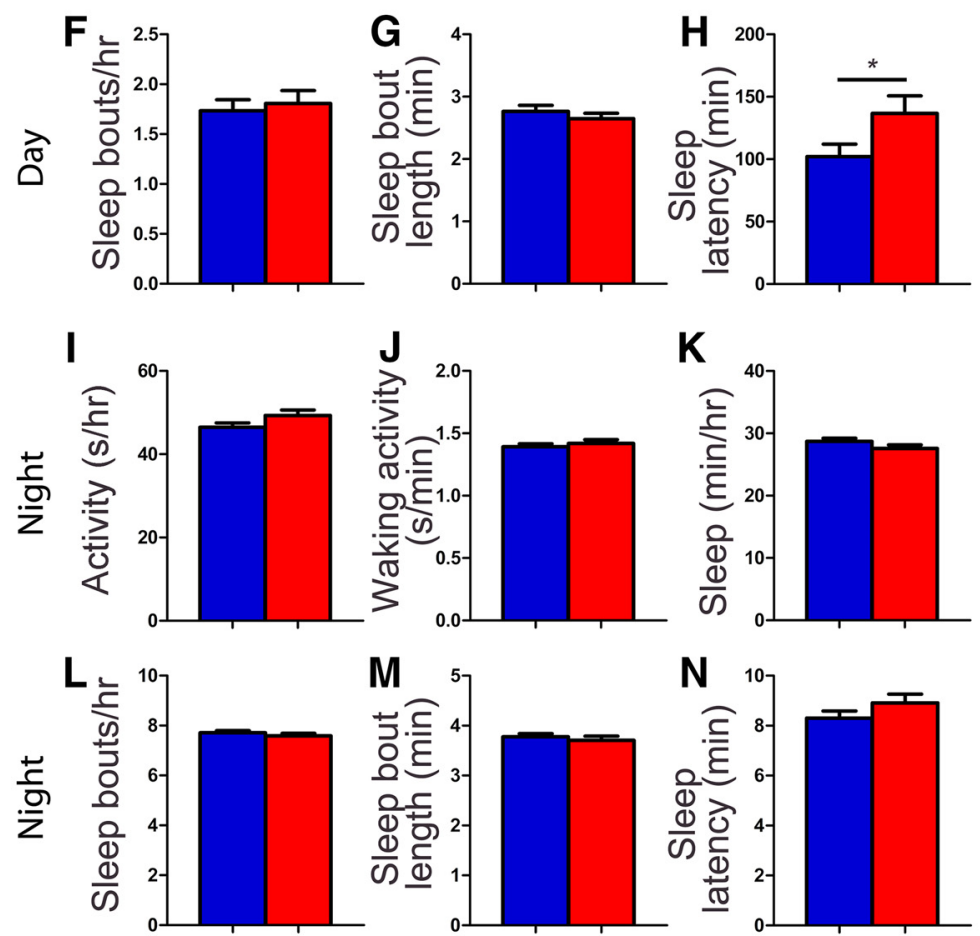

Figure 3. The absence of a larval hdc zygotic mutant behavioral phenotype is not due to rescue by maternal WT hdc. hdc-/- (red) and $h d c+/-$ (blue) larvae that were generated by mating an $h d c-/-$ female to an $h d c+/-$ male fish do not show major changes in measured sleep/wake behaviors, except that $h d c-/-$ larvae have a longer latency to first sleep bout during the day $(\boldsymbol{H})$. Line and bar graphs represent the mean \pm SEM for five experiments combined; $n$ indicates the number of animals analyzed. $* p<0.05$ for the indicated comparison by Student's $t$ test. 


\title{
A
}

VGNLYIVSLSVADLIVGAVVMPMN I LYLLMSKWSLGRPLCLFWLSMDYVASTASIFSVFILCIDRYRSVQQPLRYLKYRTKTRA VGNLYIVSLSVADLIVGAVVMPMN I LYLIMTKWSLGRPLCLFWLSMDYVASTASIFSVFILCIDRYRSVQQPLRYLRYRTKTRA VGNLYIVSLSIADLIVGATVMPLNLVYLLEDQWKLGHVVCQFWLVMDYVASTASIFSLFILCLDRYRSVRHPLQYLKYRTRGRA Hrh1_Drhu3427 VGN L Y IVSLSIADLIVGATVMPLNLVYLLEDQWKLGHVVCQFWLVMDYVASTASIFSLFILCLDRYRSVRHPLQYLKYRTRGRA

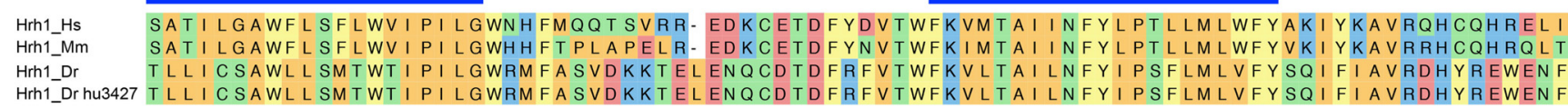

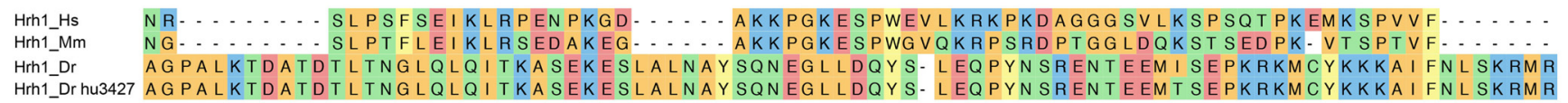

\begin{abstract}
Hrh1_Hs - DSDTTTETAPGKGKLRSGSNTGLDYIKFTWKRLRSHSRQYVSGLHMNRERKAAKQLGFIMAAFILCWIPYFIFFMVIAFCKNC Hrh1_Mm TDSDTSIEPGLGKVKARSRSNSGLDYIKVTWKRLRSHSRQYVSGLHLNRERKAAKQLGCIMAAFILCWIPYFIFFMVIAFCNSC Hrh1_Dr LCTPPSPPSPWAENSPPLDASIALP-AKQSWQKLCEQSKQSIHSMRIRKERKAARQLGFIIGVFMVCWIPYFITFMVMALCETC

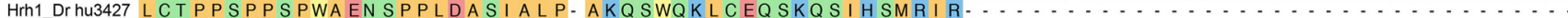

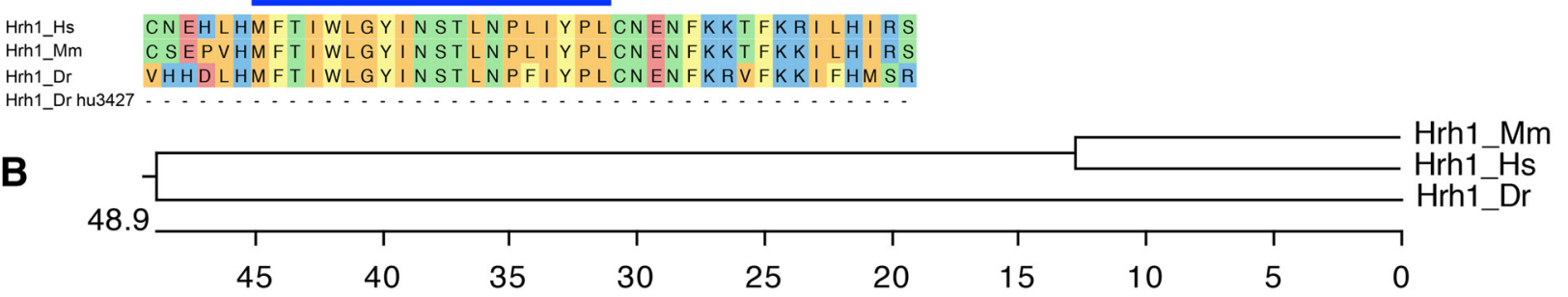

Figure 4. Alignment of Hrh1. A, Hrh1 protein sequences from human (Hs, ENSG00000196639), mouse (Mm, ENSMUSG00000053004), zebrafish WT (Dr, ENSDARG00000052425), and zebrafish mutant (Dr hu3427). Blue lines indicate predicted transmembrane domains. Amino acids are colored to indicate residues with similar properties. $\boldsymbol{B}$, Phylogenetic tree of human, mouse, and zebrafish Hrh1. Values indicate the number of amino acid substitutions per 100 residues.

Parmentier et al., 2002; Anaclet et al., 2009), we challenged animals using two paradigms. First, we exposed larvae to alternating 1-h periods of light and darkness for $24 \mathrm{~h}$ (Fig. 6). As previously shown, WT larvae exhibit a brief startle response to lights on, followed by a gradual increase in locomotor activity levels that reach a plateau, followed by a strong locomotor response when the lights are turned off (Prober et al., 2006; Burgess and Granato, 2007). We observed no significant differences in behavioral response to either light transition, or in total amount of locomotor activity during the light or dark periods, for hdc-/- or hrh1-/- animals compared with sibling controls (Fig. 6). This contrasts with a previous study, in which larvae injected with an hdc-specific morpholino, or treated with the Hdc inhibitor $\alpha$-fluoromethylhistidine ( $\alpha$ $\mathrm{FMH}$ ) or the Hrh1 antagonist pyrilamine, failed to respond to a lights off stimulus (Sundvik et al., 2011). Second, we subjected larvae to a mechano-acoustic stimulus of varying intensity every minute for $7.5 \mathrm{~h}$ during the day or night. We previously showed that larvae exhibit a brief startle response in a manner that depends on stimulus intensity and that does not habituate under these conditions. We observed no difference in the response of $h d c-/-$ or hrh1-/- animals compared with sibling controls (Fig. $7 A, B, D, E)$. We also performed a similar assay during the night using a 5-min intertrial interval and a single stimulus intensity (Fig. 7C,F). Since sleep in zebrafish larvae is defined as one or more minutes of inactivity, by allowing 5 min between trials, we could assess the response of specifically sleeping larvae and thus determine whether arousal from the sleep state is affected by mutation of $h d c$ or $h r h 1$. We found that sleeping mutant larvae and sibling 

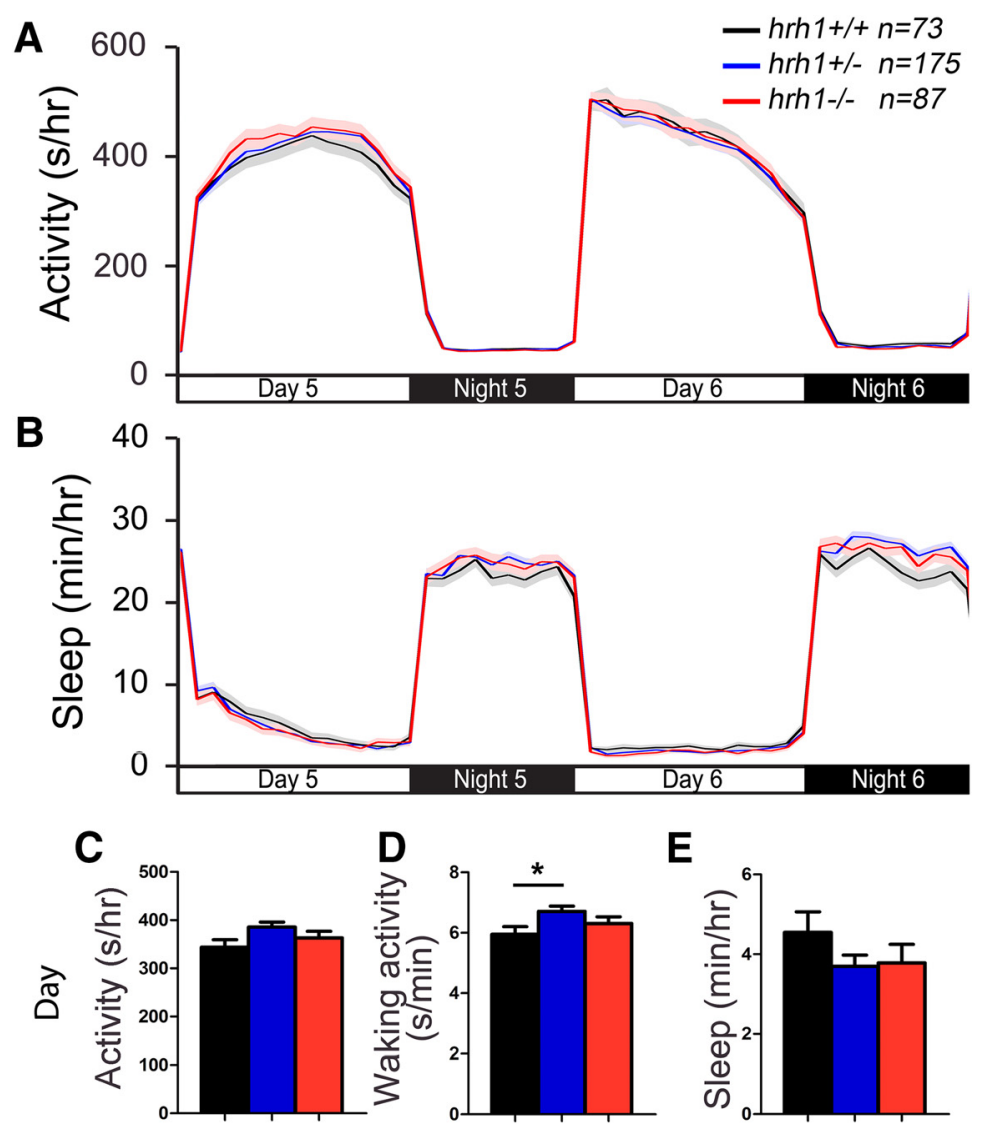

E
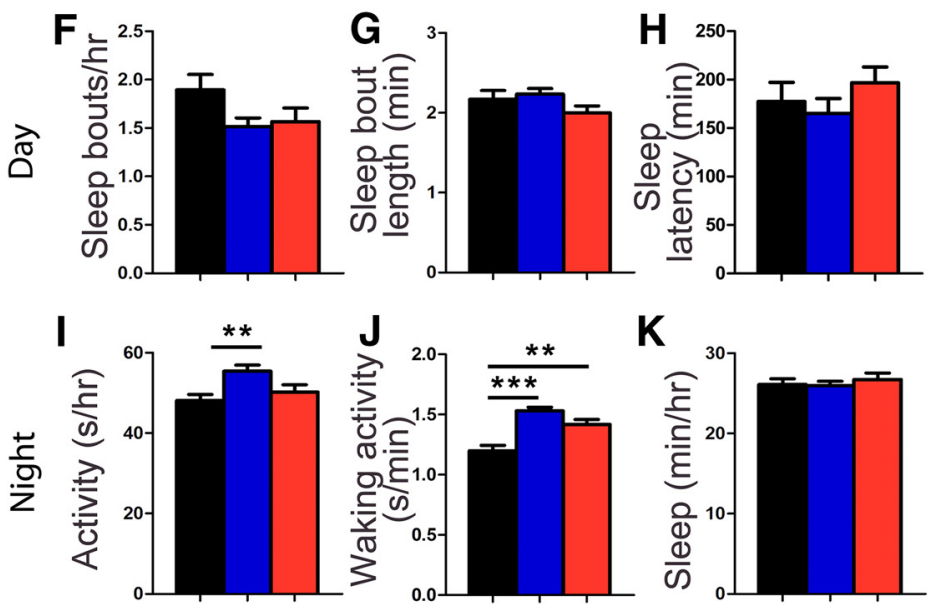

$\mathbf{K}$
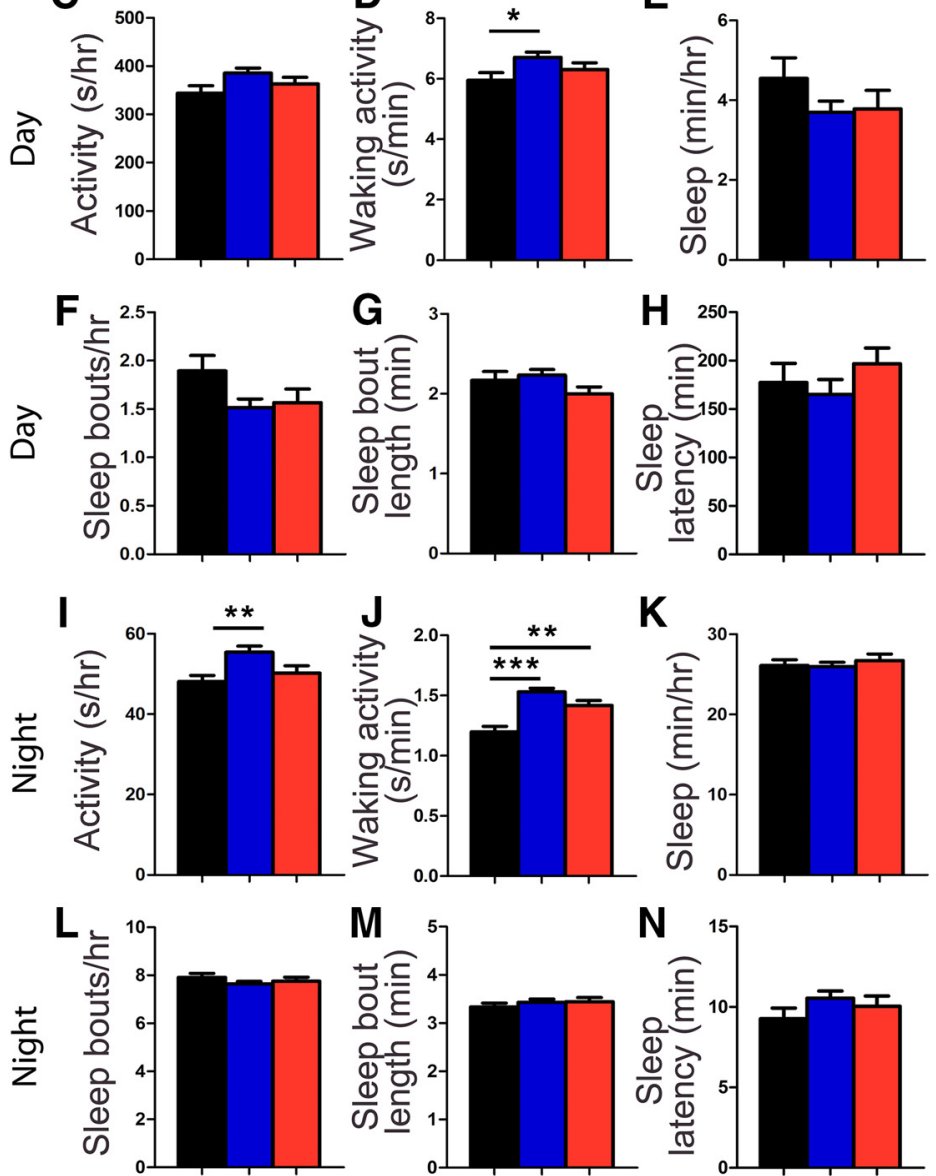

Figure 5. hrh1 mutant larvae exhibit largely normal sleep/wake behaviors. $h r h 1-/-$ (red), $h r h 1+/-$ (blue), and $h r h 1+/+$ (black) siblings exhibit similar amounts of all measured parameters, except that $h r h 1-/-$ and $h r h 1+/-$ larvae exhibit higher waking activity at night compared with $h r h 1+/+$ siblings $(\boldsymbol{J})$, and $h r h 1+/-$ larvae exhibit higher waking activity during the day $(\boldsymbol{D})$ and higher activity at night (I) compared with their $h r h 1+/+$ siblings. Line and bar graphs represent the mean \pm SEM for four experiments combined; $n$ indicates the number of animals analyzed. $* p<0.05 ; * * p<0.01 ; * * * p<0.001$ for the indicated comparisons by one-way ANOVA with Tukey's HSD test. 

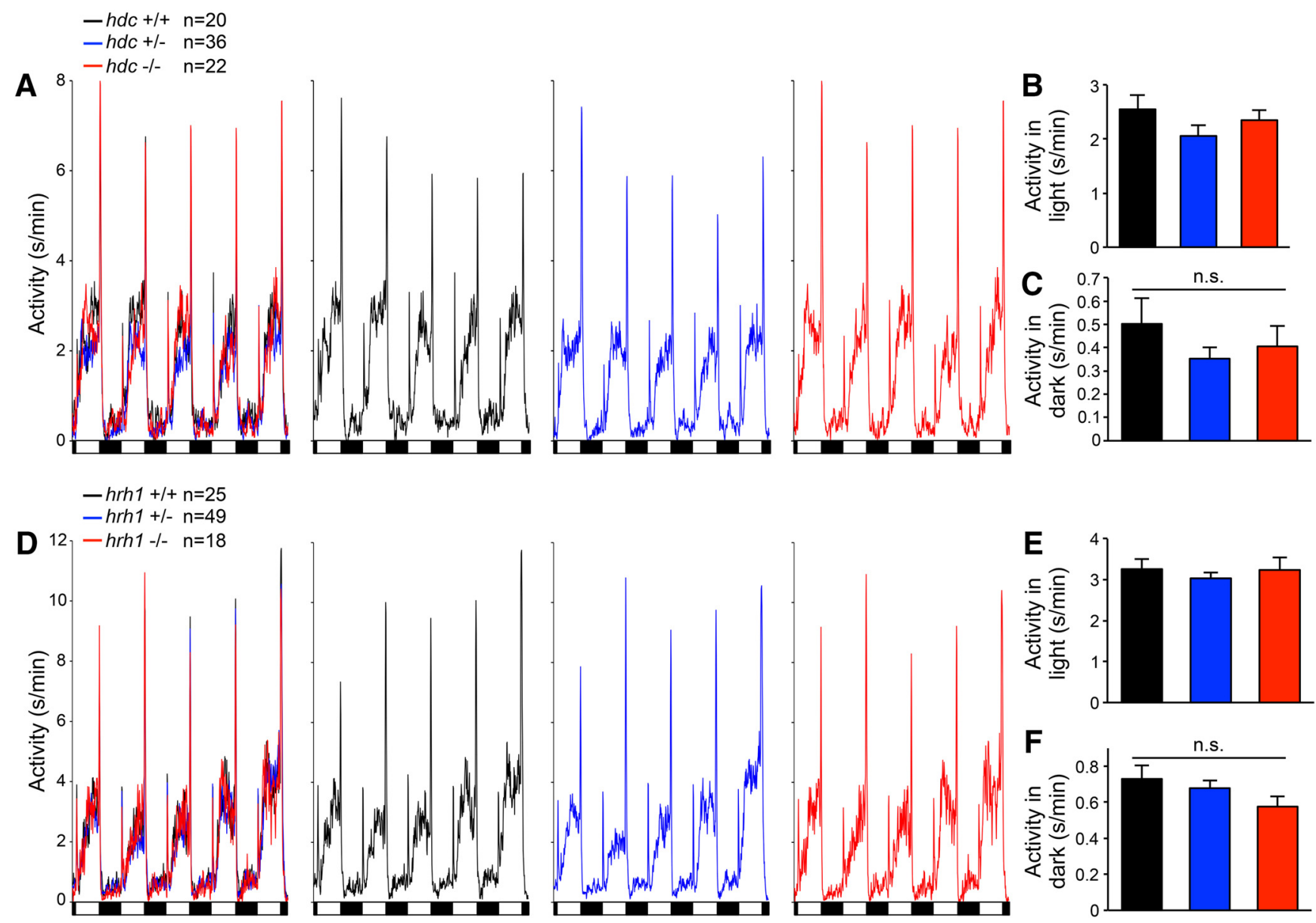

Figure 6. hdc and $h r h 1$ mutants exhibit normal behavioral responses to alternating periods of light and darkness. $\boldsymbol{A}, \boldsymbol{D}$, Line graphs represent the mean locomotor activity of $h d c+/+$ (black), $h d c+/-$ (blue), and $h d c-/-$ (red) larvae $(\boldsymbol{A})$, or $h r h 1+/+($ black), $h r h 1+/-$ (blue), and $h r h 1-/-($ red) larvae $(\boldsymbol{D})$, plotted in a single graph containing all three genotypes (left) as well as in graphs showing individual genotypes to facilitate comparisons. Similar behavioral responses to light onset and offset are apparent for $h d c-/-(\boldsymbol{A})$ and hrh1-/- (D) larvae compared with sibling controls. Black and white boxes indicate 1-h periods of light and darkness. $\boldsymbol{B}, \boldsymbol{C}, \boldsymbol{E}, \boldsymbol{F}$, Bar graphs represent the mean \pm SEM locomotor activity during light and dark periods. There is no significant difference in the amount of locomotor activity during either light or dark periods for $h d c-/-(\boldsymbol{B}, \boldsymbol{C})$ or $h r h 1-/-(\boldsymbol{E}, \boldsymbol{F})$ larvae compared with sibling controls. Data for five light/dark cycles at night is shown, but similar results are obtained when data are averaged over the entire 24-h experiment; $n$ indicates the number of animals analyzed. n.s. = not significant $(p>0.05)$ for all comparisons by one-way ANOVA with Tukey's HSD test.

controls were equally responsive to the stimulus. We conclude that $h d c$ and $h r h 1$ mutants exhibit normal behavioral responses to these environmental perturbations, as well as normal sleep depth.

\section{hrh2 mutant larvae lack sleep/wake phenotypes}

It is unclear whether Hrh2 signaling is involved in sleep/ wake regulation. Systemic administration of Hrh2 agonists and antagonists has no effect on sleep or wake levels in nocturnal rodents (Monti et al., 1986; Monti et al., 1990), but application of an Hrh2 agonist in the preoptic region of the brain promotes wakefulness in cats (Lin et al., 1994; Lin et al., 1996). To determine whether signaling through Hrh2 is required for normal sleep/wake behaviors, we generated mutations in the two zebrafish hrh2 paralogs, hrh2a and hrh2b (Fig. 8). Both mutants contain early stop codons and generate predicted proteins that lack several transmembrane domains and should thus be non- functional. To assay these mutants for sleep phenotypes, we compared the behavior of larvae generated from an incross of $h r h 2 a+/-; h r h 2 b+/-$ animals. We found that the behavior of $h r h 2 a-/-; h r h 2 b-/-$ larvae was indistinguishable from their hrh2a+/-; hrh2b+/- siblings (Fig. 9). Thus, mutation of zebrafish Hrh2 receptors does not result in detectable sleep/wake phenotypes.

\section{hrh3 mutant larvae do not show major changes in sleep/wake behaviors}

Hrh3 is thought to act as an autoreceptor (Morisset et al., 2000; Arrang et al., 2007) that inhibits histamine release from histaminergic neurons, and as a presynaptic heteroreceptor on nonhistaminergic neurons to control the release of other neurotransmitters, including acetylcholine, GABA and glutamate (Schlicker et al., 1994; Haas et al., 2008; Samaranayake et al., 2016). Thus, loss of Hrh3 autoreceptors is expected to increase histamine 

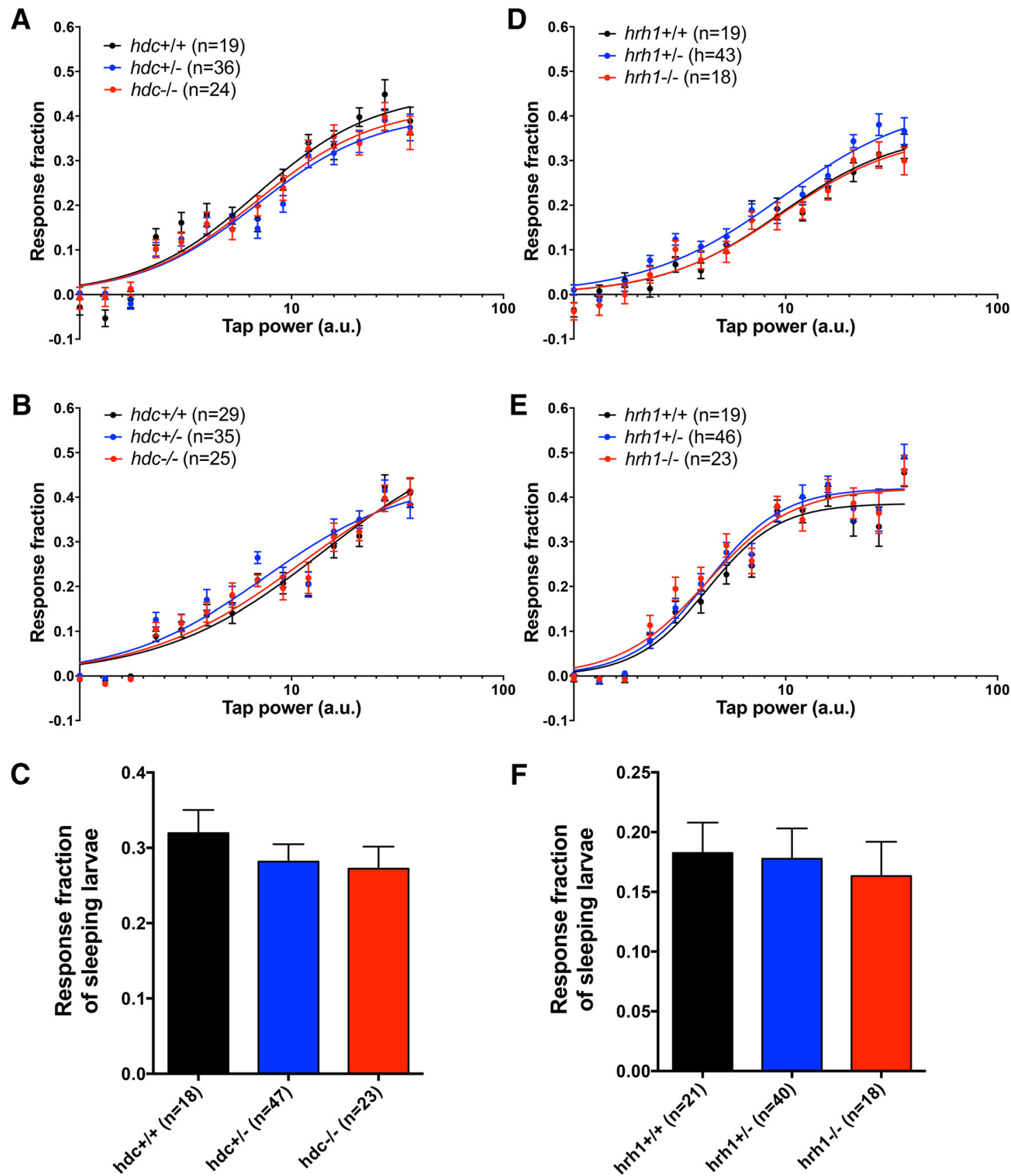

Figure 7. hdc and $h r h 1$ mutants exhibit normal behavioral responses to a mechano-acoustic stimulus. $h d c+/+, h d c+/-$, and $h d c-/-$ larvae have the same response profile during the day $(\boldsymbol{A})$ and night $(\boldsymbol{B})(\boldsymbol{p}>0.05$, extra sum-of-squares $F$ test), and are equally likely to respond to tapping when asleep $(\boldsymbol{C})(p>0.05$, one-way ANOVA). Similar results were obtained for $h r h 1+/+, h r h 1+/-$, and $h r h 1-/-$ larvae $(\boldsymbol{D}-\boldsymbol{F})$. Bar graphs represent the mean \pm SEM; $n$ indicates the number of animals analyzed.

release, and thus suppress sleep and increase wakefulness, consistent with studies using Hrh3 antagonists (Parmentier et al., 2002; Huang et al., 2006; Parmentier et al., 2007; Lin et al., 2008), but inconsistent with the hypoactivity and increased sleep observed in hrh3-/- mice (Toyota et al., 2002; Gondard et al., 2013). To assess the role of Hrh3 in zebrafish sleep, we generated an hrh3 mutant that encodes for a protein that is predicted to lack two transmembrane domains and should, thus, be nonfunctional (Fig. 10). We found that $h r h 3-/-$ larvae have essentially normal sleep/wake behaviors compared with their $h r h 3+/-$ and $h r h 3+/+$ siblings (Fig. 11). In contrast to $h r h 3-/-$ mice, which show fragmented sleep during the dark phase (Gondard et al., 2013), we found that $h r h 3-/$ - larvae have slightly longer sleep bouts during both the day and night (Fig. $11 G, M ; 11 \%$ and $12 \%$ increase during day and night, respectively, $p<0.05$ by Tukey's HSD test). Despite this increase in sleep bout length, the total amount of sleep in zebrafish hrh3-/- larvae is statistically indistinguishable from sibling controls (Fig. 11B,E,K), although hrh3-/- lar- 


\section{A}

Hrh2_Hs $\mathrm{Hhr} 2 \mathrm{Mm}$

Hhr2a Dr

Hhr2a_Drd

Hrh2b_Dr

Hrh2b_Drd8
MAPNGTASSFCLDSTACKITITVVLAVLILITVAGNVVVCLAVGLNRRLRNLTNCFIVSLA|TDLLLGLLVLPFSA I YQL - SCKWS MEPNGTVHSCCLDSIALKVTISVVLTTLIFITVAGNVVVCLAVSLNRRLRSLTNCFIVSLAATDLLLGLLVMPFSA I YQL-SFKWS $\ldots \ldots \ldots$..............................

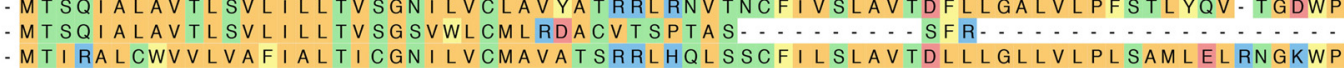
- MT I RALCWVVLVAFIALTICGN ILVCMAVATSRRLHQLSSCFILSLAVTDLLLGTATFCHAGTAQWKVASWG
Hrh2_Hs $\mathrm{Hhr} 2 \mathrm{Mm}$

Hhr2a_Dr

Hhr2a_Drd10 Hrh2b_Dr Hrh2b_Drd8

FGKVFCN I YTSLDVMLCTAS I LNLFM I SLDRYCAVMDPLRYPVLVTPVRVA I SLVLIWVISITLSFLSI HLGWNSRNETSK. - - FGQVFCN I YTSLDVMLCTASILNLFMISLDRYCAVTDPLRYPVLVTPVRVAISLVFIWVISITLSFLSIHLGWNSRNGTRG- - LGAHFCNI YI SLDVMLCTASILNLFA I SLDRYFAVTAPLRYPMLVLPWRVGA ILATIWLVSVGVSFVPI HLGWNTRDLSVQNI - -

LGGVFCN I Y I SMDVMLSSA SILTLLA I SVDRYLA I SNPLFYPRRVTPRRVA I ALTA I WTCSLAVSFVSINLGWNSPDFRVQNLDWS

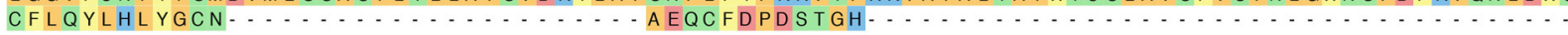

Hrh2_Hs Hhr2_Mm Hhr2a_Dr

Hhr2a_Drd 10 Hrh2b_Dr Hrh2b_Drd8
- . - GNHTTSKCKVQVNEVYGLVDGLVTFYLPLLIMC I TYYRIFKVARDQAKRINHIS . . . REGDHARDCRFELNPTYA IVDAFSTFYLPLLAMCWSYHRVFRIARMRAKRI I STRRGSTSSQGVTILTLREHKATVTLAVVLGA MWDEGEEGRTCRYEWNNNYVLLKAFGIFYLPLLVMCGMYHRIFCVAREQVRRIRAATPSSA-QVANAAATAREHKATVTLAAVLGA
$\mathrm{Hrh} 2 \mathrm{Hs}$ Hhr2_Mm Hhr2a_Dr Hhr2a_Drd10 Hrh2bDr Hrh2b Drd8

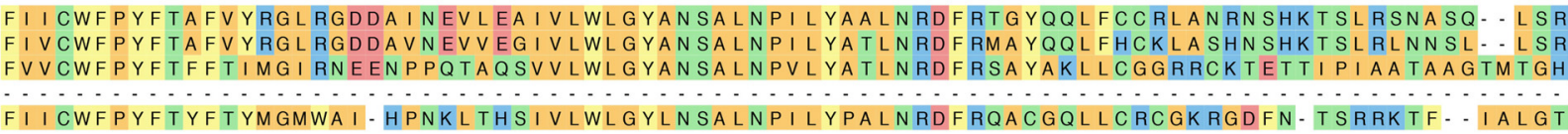

FI I CWFPYFTAFVYRGLRGDDA INEVLEA IVLWLGYANSALNPILYAALNRDFRTGYQQLFCCRLANRNSHKTSLRSNASQ- - LSR FIVCWFPYFTAFVYRGLRGDDAVNEVVEGIVLWLGYANSALNPILYATLNRDFRMAYQQLFHCKLASHNSHKTSLRLNNSL. - LSR FVVCWFPYFTFFTIMGI RNEENPPQTAQSVVLWLGYANSALNPVLYATLNRDFRSAYAKLLCGGRRCKTETTIPIAATAAGTMTGH FI I CWFPYFTYFTYMGMWA I- HPNKLTHSIVLWLGYLNSALNPILYYPALNRDFRQACGQLLCRCGKRGDFN-TSRRKTF- - IALGT

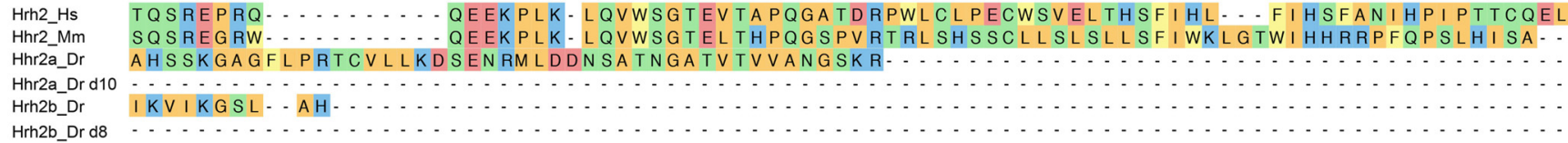

B

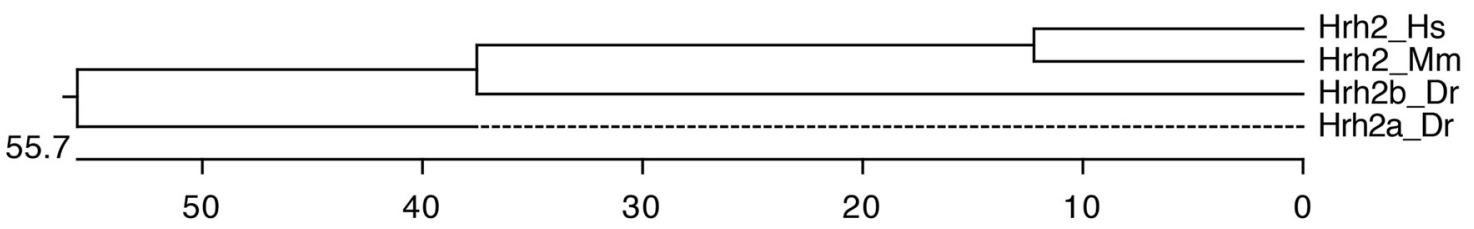

Figure 8. Alignment of Hrh2. A, Hrh2 protein sequences from human (Hs, ENSG00000113749), mouse (Mm, ENSMUSG00000034987), zebrafish WT (Hrh2a_Dr, NM_001045338; Hrh2b_Dr, ENSDARG00000057479), and zebrafish mutant (Hrh2a_Dr d10; Hrh2b_Dr d8). Blue lines indicate predicted transmembrane domains. Amino acids are colored to indicate residues with similar properties. B, Phylogenetic tree of human, mouse, and zebrafish Hrh2. Values indicate the number of amino acid substitutions per 100 residues.

vae show a trend of more daytime sleep (Fig. 11E) consistent with the increased nighttime sleep of nocturnal hrh3-/mice (Gondard et al., 2013).

\section{hrh1; hrh2a; hrh2b; hrh3 quadruple mutant larvae lack sleep-wake phenotypes}

To determine whether the lack of robust sleep/wake phenotypes in zebrafish Hrh mutants is due to functional redundancy, we generated and tested $h r h 1 ; h r h 2 a ; h r h 2 b$; hrh3 quadruple mutant larvae. Comparison of hrh1-/-; hrh2a+l-; hrh2b+/-; hrh3-l- larvae to hrh1-/-; hrh2a-I-; hrh2b-/-; hrh3-/- siblings revealed no differences in sleep/wake behaviors (Fig. 12).

\section{hdc and hrh1 are not required for Hcrt-induced arousal in zebrafish larvae}

The mammalian TMN receives excitatory input from Hcrt neurons (Peyron et al., 1998; Eriksson et al., 2001;
Torrealba et al., 2003; Schöne et al., 2012), and wakefulness induced by Hcrt infusion is blocked in hrh1 knockout mice (Huang et al., 2001) and by the Hrh1 antagonist pyrilamine in rats (Yamanaka et al., 2002; Shigemoto et al., 2004), suggesting that Hrh1 is required for Hcrtinduced arousal. However, others have shown that Hrh1 is not required for normal Hcrt function in rodents (Hondo et al., 2010), and arousal induced by optogenetic stimulation of Hcrt neurons is unaffected in hdc mutant mice (Carter et al., 2009). To test whether histamine is required for Hcrt-induced arousal in zebrafish, we compared the effect of Hcrt overexpression in $h d c+/-$ larvae to their hdc-/- siblings. Consistent with a previous study (Prober et al., 2006), heat shock-induced Hcrt overexpression of Tg(hsp:Hcrt); hdc+/- larvae increased locomotor activity and waking activity, decreased sleep and the number of sleep bouts, and increased sleep latency at night (Fig. 13). These phenotypes were statistically indistinguishable from 

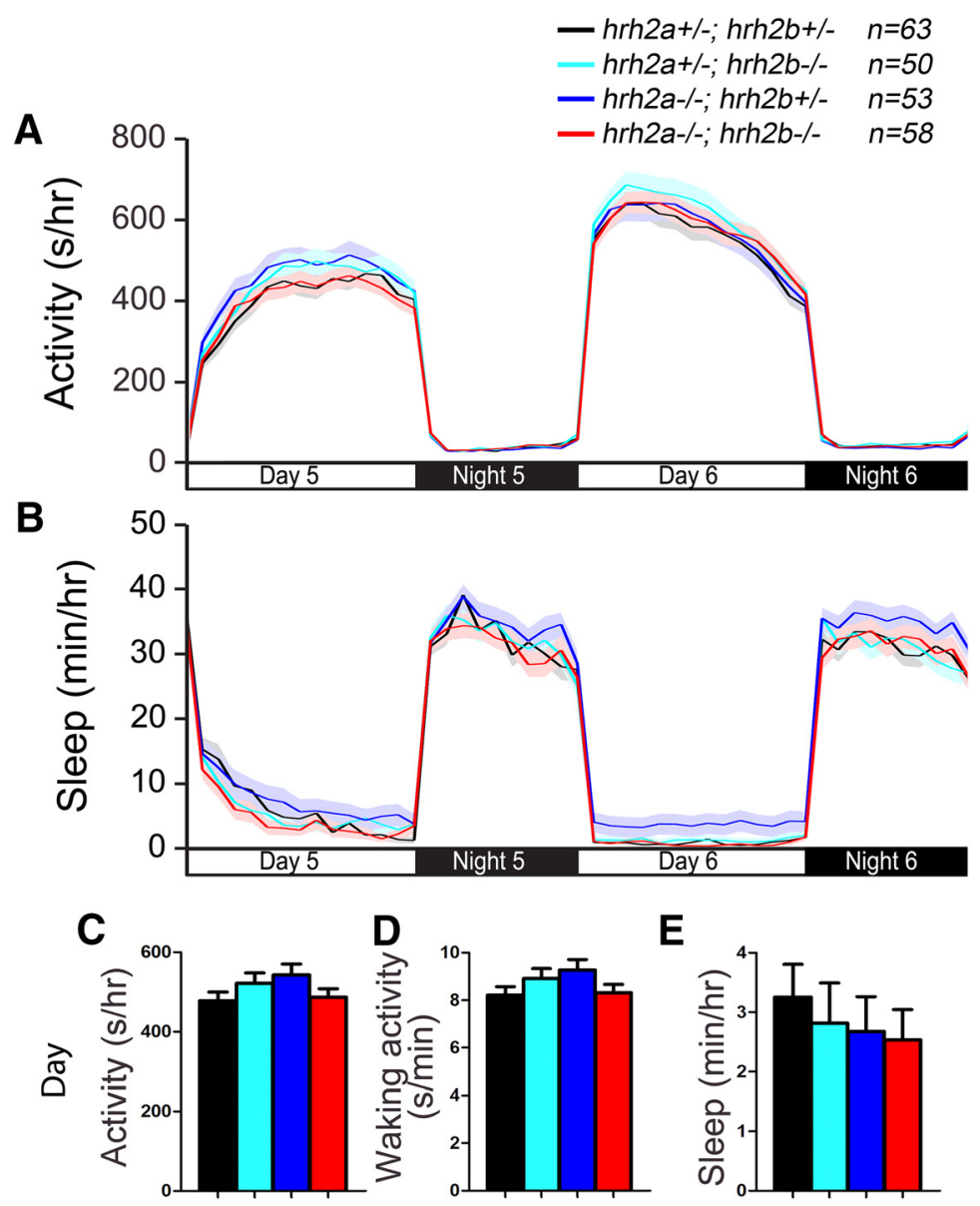

E
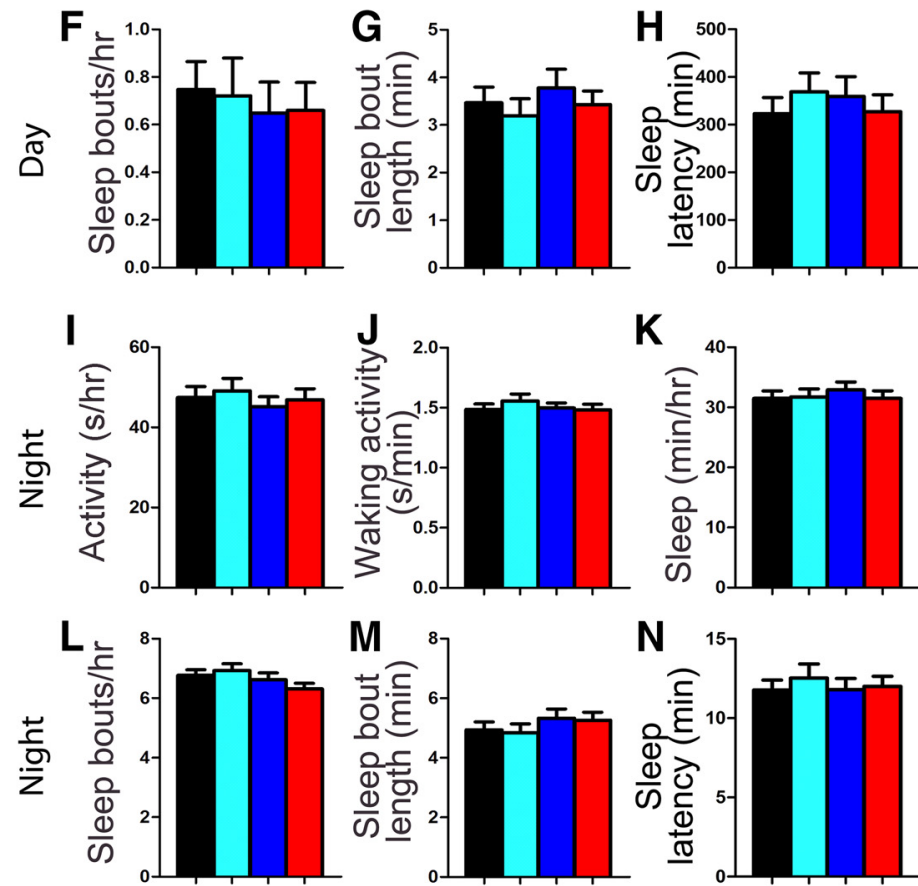

Figure 9. hrh2 mutant larvae exhibit normal sleep/wake behaviors. hrh2a-/-; hrh2b-/- (red), hrh2a+/-; hrh2b+/- (black), $h r h 2 a+/-; h r h 2 b-/-$ (cyan), and $h r h 2 a-/-; h r h 2 b+/-$ (blue) larvae exhibit similar amounts of all measured sleep/wake parameters. Line and bar graphs represent the mean \pm SEM for three experiments combined; $n$ indicates the number of animals analyzed. $p>$ 0.05 for all comparisons by one-way ANOVA with Tukey's HSD test. 


\section{A}

Hrh3_Hs Hhr3_Mm

Hhr3 Dr

MOSSLISVAHSPG PT AVSLMWK SGNHOSSNWSGMER

FA AWTAVLAALMALLIVATVLGNAL $\begin{array}{lll}\text { Hrh3_Hs } & \text { VMLAFVADSSLRTQNNFFLLNLA I SDFLVGAFCI PLYVPYVLTGRWTFGRGLCKLWLVVDYLLCTSSAFN IVLISYDRFLSVTR } \\ \text { Hhr3_Mm } & \text { VMLAFVADSSLRTQNNFFLLNLA I SDFLVGAFCI PLYVPYVLTGRWTFGRGLCKLWLVVDYLLCASSVFN IVLISYDRFLSVTR } \\ \text { Hhr3_Dr } & \text { VILAFVVEKSLRTQGNFFFLNLA I ADFLVGGFCI PVYIPYVLTGEWRLGRGLCKLWLVVDYMLCTASVFN IVLISFDRFQSVTK } \\ \text { Hhr3_Drd4 VILAFVVEKSLRTQGNFFFLNLA I DDFLVGGFCI PVYI PYVLTGEWRLGRGLCKLWLVVDYMLCTASVFNIVLISFDRFQSVTK }\end{array}$

Hrh3_Hs AVSYRAQQGDTRRAVRKMLLVWVLAFLLYGPAILSWEYLSGGSSI PEGHCYAEFFYNWYFLITASTLEFFTPFLSVTFFNLSIY Hhr3_Mm AVSYRAQQGDTRRAVRKMALVWVLAFLLYGPAILSWEYLSGGSSIPEGHCYAEFFYNWYFLITASTLEFFTPFLSVTFFNLSIY Hhr3 Dr AVSYRCQKG।TKDAVLKMLCVWLAAFLLYGPA I I SWEHITGGSVVPDGECYAEFYFNWYFLMTASTVEFFTPFI SVTYFNLSIY Hhr3_Drd4 AVSYRCQKGITKDAVLKMLCVWLAAFLLYGPAI I SWEHITGGSVVPDGECYAEFYFNWYFLMTASTVEFFTPFISVTYFNLSI Y Hhr3 Lm L I QRRTRLRLDGAREAAGPEPPPEAQPSPPP-PPGCWGCWQKGHGEAMPLHRYGVGEAAVGAEAGEATLGGGGGGGSVASPTS LN I QRRTRLRLDGGRE- AGPEPPPDAQPSPPPAPPSCWGCWPKGHGEAMPLHRYGVGEAGPGVETGEAGLGGGSGGGAAASPT

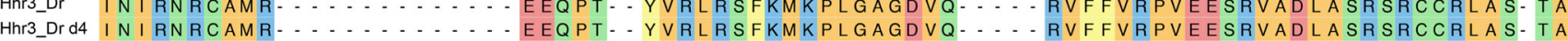

Hrh3_Hs SSGSSSRGTERPRSLKRGSKPSASSASLEKRMKMVSQ-

SFTQRFRLSRDRKVAKSLAVIVSIFG Hhr3_Mm SSGSSSRGTERPRSLKRGSKPSASSASLEKRMKMVSQ. SI TQRFRLSRDKKVAKSLAIIVSIFG Hhr3_Dr KVSAAEFGNGRQSKRRDSTLADLPPLQVEERILAASEAQFHYVDHSAGPHRHRPDMVASLANRFRLSRDKKVAKSLAVIVCVFG Hhr3_Drd4 KVSAAEFGNGRQSKRRDSTLADLPPLQKS

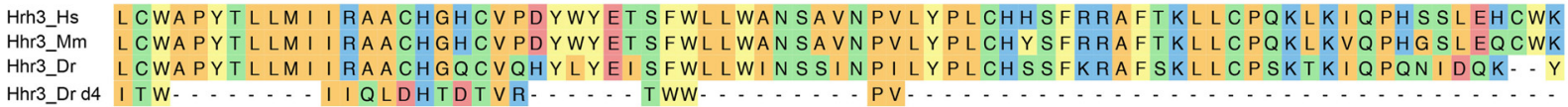

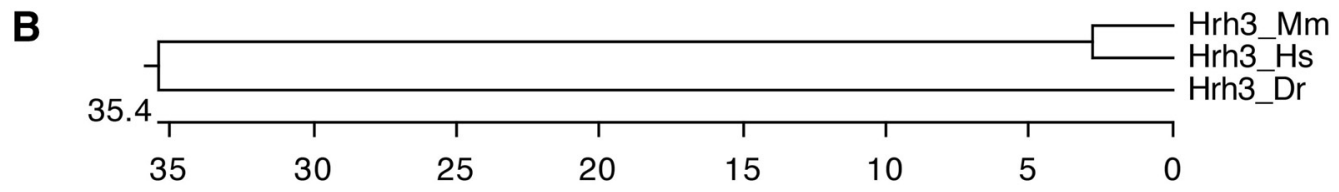

Figure 10. Alignment of Hrh3. A, Hrh3 protein sequences from human (Hs, ENSG00000101180), mouse (Mm, ENSMUSG00000039059), zebrafish WT (Dr, ENSDARG00000035942), and zebrafish mutant (Dr d4). Blue lines indicate predicted transmembrane domains. Amino acids are colored to indicate residues with similar properties. $\boldsymbol{B}$, Phylogenetic tree of human, mouse, and zebrafish Hrh3. Values indicate the number of amino acid substitutions per 100 residues.

Tg(hsp:Hcrt); hdc-/- larvae (Fig. 13), except that Tg(hsp: Hcrt); hdc-l- larvae had slightly shorter sleep bouts during the night after heat shock than Tg(hsp:Hcrt); $h d c+/-$ larvae (Fig. 13M'). The latter phenotype suggests that the ability of Hcrt overexpression to decrease sleep bout length (Prober et al., 2006) is enhanced in histamine deficient animals, possibly resulting from compensatory changes in sleep/ wake neuronal circuits that mediate Hcrt-induced arousal in hdc $-/-$ animals. However, overall, these results suggest that $h d c$ is not required for Hcrt overexpression-induced arousal in zebrafish larvae.

To test whether Hrh1 is required for Hcrt-induced arousal in zebrafish, we compared the effect of Hcrt overexpression in $h r h 1+/-$ larvae to their $h r h 1-/-$ siblings. Hcrt overexpression in Tg(hsp:Hcrt); $h r h 1+/-$ larvae increased locomotor activity and waking activity, decreased sleep and the number of sleep bouts, and increased sleep latency at night (Fig. 14). Similar to the Tg(hsp:Hcrt); hdc-l- result, the behavior of Tg(hsp:Hcrt); hrh1-/larvae was indistinguishable from their Tg(hsp:Hcrt); hrh1+/- siblings (Fig. 14), except that Hcrt overexpression failed to increase sleep latency at night in $h r h 1+/-$ animals but not in their hrh $1-/-$ siblings (Fig. 14N').
Overall, these results indicate that $h r h 1$ mutant zebrafish larvae exhibit normal responses to Hcrt overexpression.

As an alternative approach to test whether histamine signaling is required for Hcrt-induced arousal, we compared the behavioral effect of stimulating hort-expressing neurons using ReaChR (Fig. 15A; Lin et al., 2013) in $h d c-/-$ larvae to their $h d c+/-$ siblings. Similar to our previous study using Tg(hcrt:ChR2) (Singh et al., 2015), we found that blue light increased locomotor activity in Tg(hcrt:ReaChR); hdc+/- larvae compared with their $h d c+/-$ siblings, as well as in Tg(hcrt:ReaChR); $h d c-/-$ larvae compared with their hdc-/- siblings (Fig. 15B,C). Importantly, there was no significant difference in the hcrt:ReaChR phenotype between hdc $+/-$ and hdc-lanimals (Fig. 15C). We obtained similar results for $h r h 1$ mutants (Fig. 15D,E). These results suggest that $h d c$ and $h r h 1$ are not required for arousal that is induced by stimulation of hcrt-expressing neurons in zebrafish larvae, consistent with a rodent study (Carter et al., 2009).

As an additional test for a functional interaction between the Hcrt and histamine systems in arousal, we examined the behavior of zebrafish larvae that lack both histamine and Hort signaling. Adult zebrafish with a 

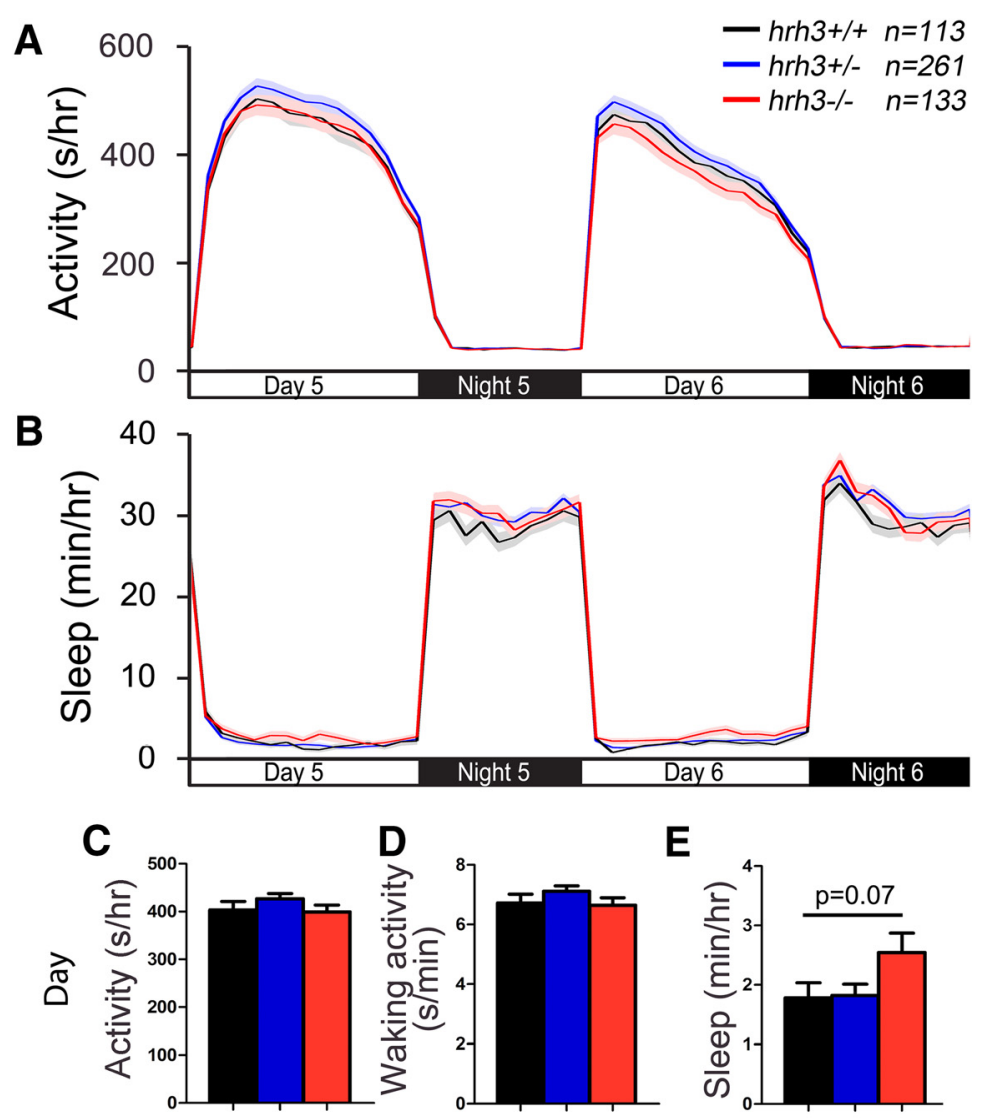

E
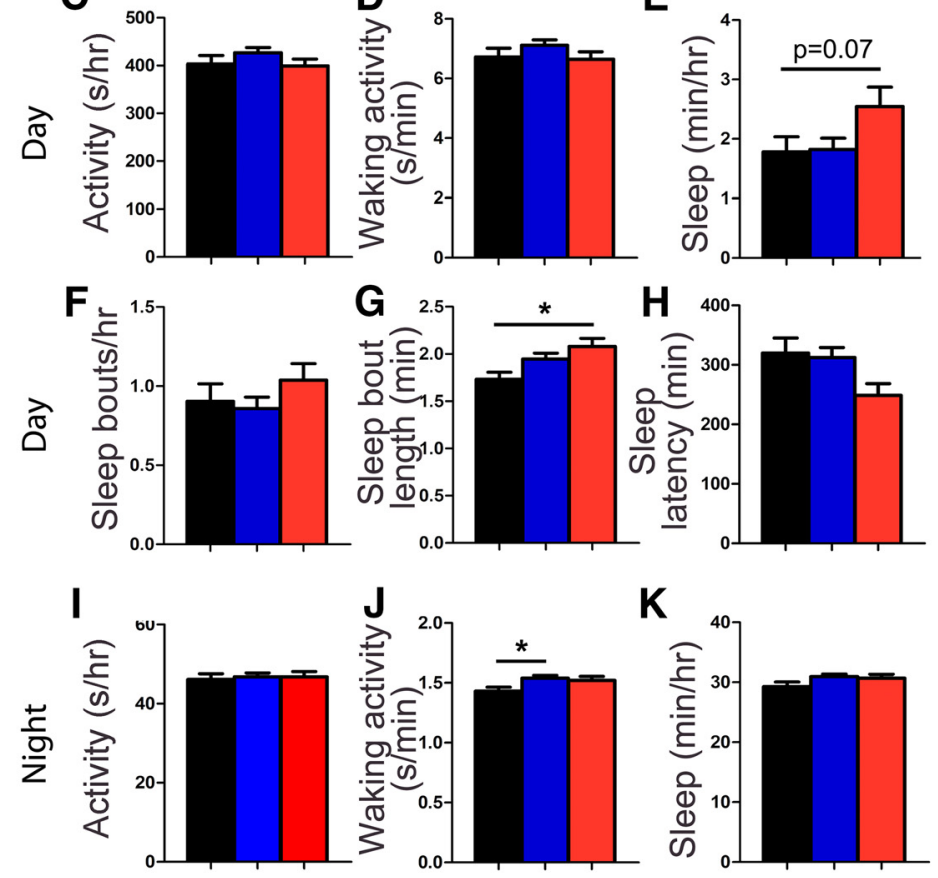

K
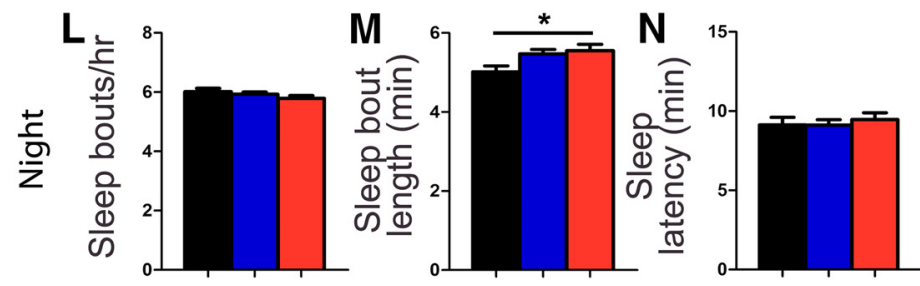

Figure 11. hrh3 mutant larvae have longer sleep bouts but otherwise exhibit normal sleep/wake behaviors. hrh3-/- (red), hrh3+/(blue), and $h r h 3+/+$ (black) larvae exhibit similar amounts of all measured parameters, except that $h r h 3-/-$ larvae have longer sleep bouts during the day and night compared with $h r h 3+/+$ siblings $(\boldsymbol{G}, \boldsymbol{M})$, and $h r h 3+/-$ larvae exhibit higher waking activity at night compared with $h r h 3+/+$ siblings $(\boldsymbol{J})$. Line and bar graphs represent the mean \pm SEM for six experiments combined; $n$ indicates the number of animals analyzed. $* p<0.05$ for the indicated comparisons by one-way ANOVA with Tukey's HSD test. 

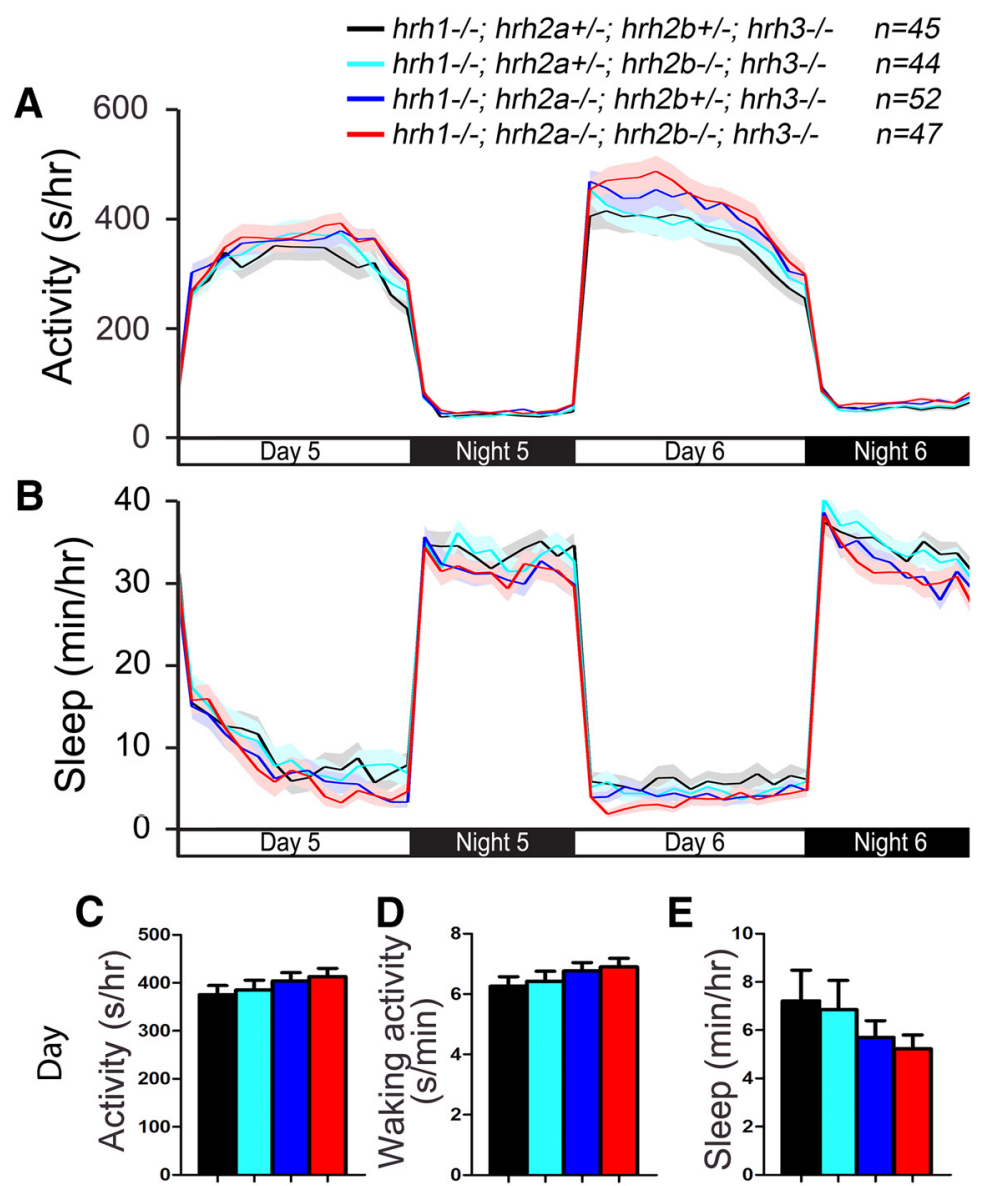

E
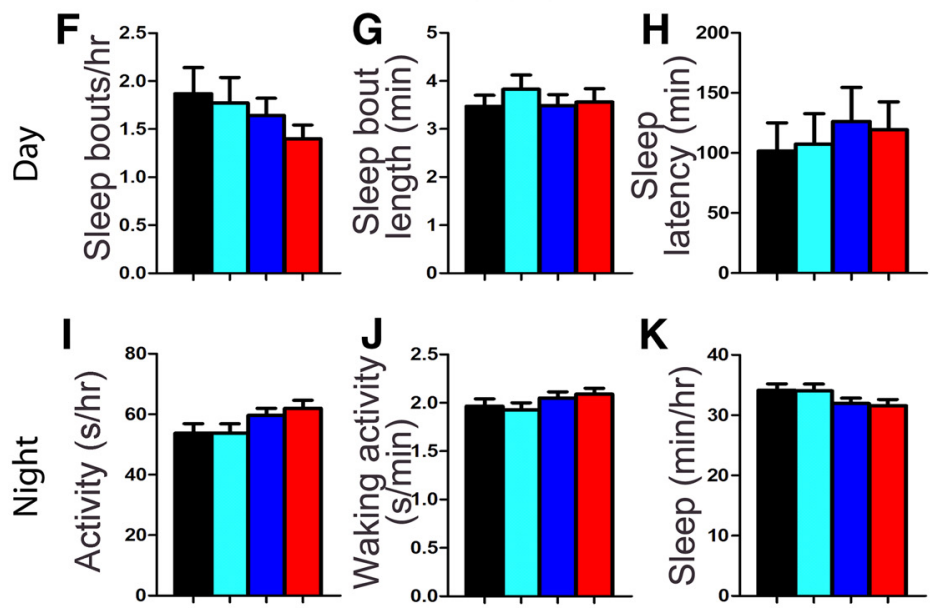

K
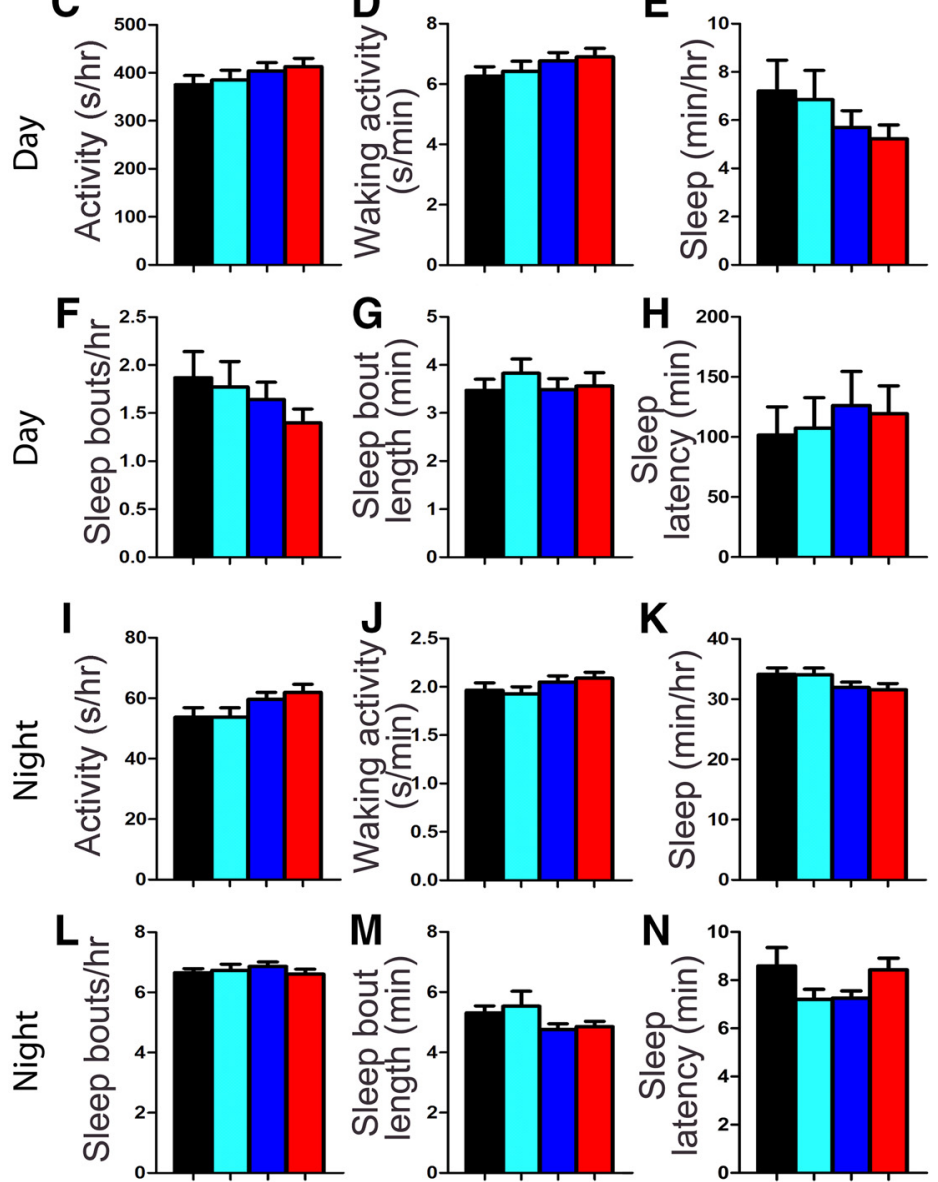

Figure 12. $h r h 1 ;$ hrh2a; $h r h 2 b ; ~ h r h 3$ quadruple mutant larvae exhibit normal sleep/wake behaviors. $h r h 1-/-; h r h 2 a-/-; h r h 2 b-/-$; hrh3-I- (red), hrh1-/-; hrh2a+/-; hrh2b+/-; hrh3-/- (black), hrh1-/-; hrh2a+/-; hrh2b-/-; hrh3-/- (cyan), and hrh1-/-; hrh2a-I-; hrh2b+l-; hrh3-/- (blue) larvae exhibit similar amounts of all measured sleep/wake parameters. Line and bar graphs represent the mean \pm SEM for two experiments combined; $n$ indicates the number of animals analyzed. $p>0.05$ for all comparisons by one-way ANOVA with Tukey's HSD test. 

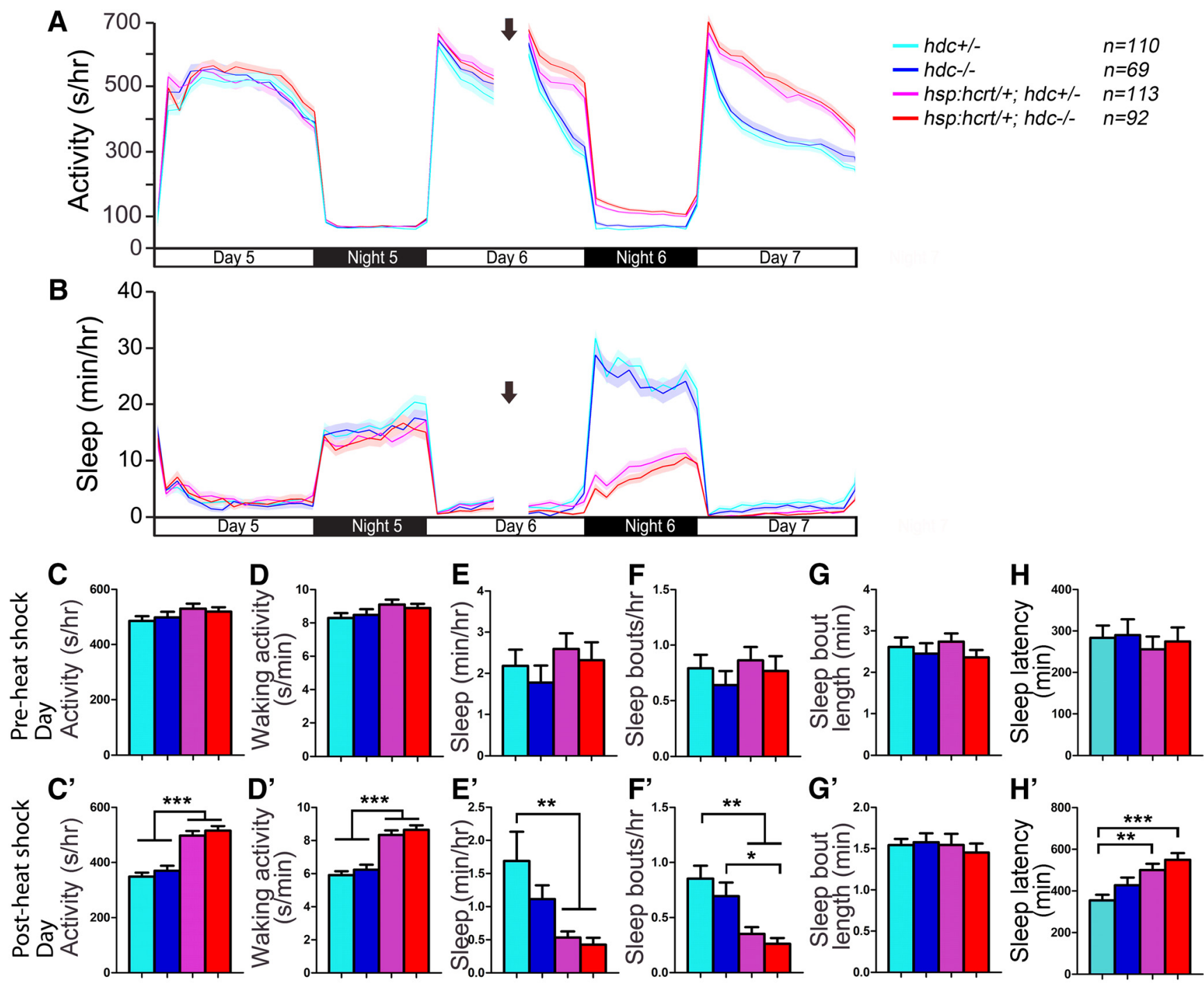

G'
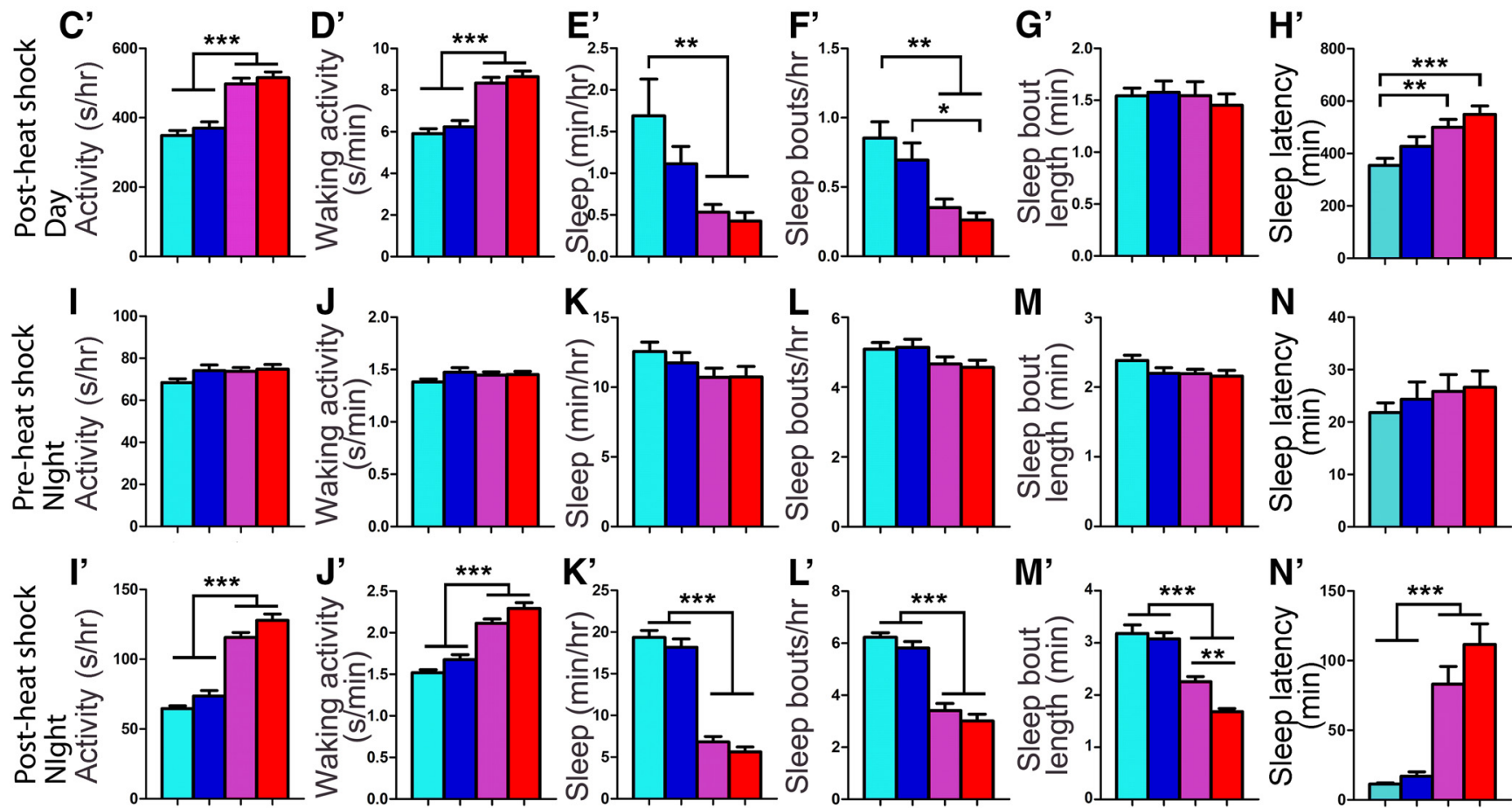

Figure 13. Hcrt overexpression-induced hyperactivity and reduced sleep does not require histamine. Before heat shock-induced Hcrt overexpression, there is no difference in all measured sleep/wake parameters between animals of each genotype $(\boldsymbol{A}-\boldsymbol{H}$, $\boldsymbol{I}-\boldsymbol{N})$. Following a 1-h heat shock during the afternoon of day 6 (indicated by arrows in line graphs), during both the day and night, both $T g(h s p: h c r t /+) ; ~ h d c-/-\left(\right.$ red) and Tg(hsp:hcrt/+); $h d c+$ - - (magenta) larvae exhibit more activity $\left(\boldsymbol{A}, \boldsymbol{C}^{\prime}, \boldsymbol{I}^{\prime}\right)$ and waking activity $\left(\boldsymbol{D}^{\prime}, \boldsymbol{J}^{\prime}\right)$, less sleep $\left(\boldsymbol{B}, \boldsymbol{E}^{\prime}, \boldsymbol{K}^{\prime}\right)$, fewer sleep bouts $\left(\boldsymbol{F}^{\prime}, \boldsymbol{L}^{\prime}\right)$, and increased latency to first sleep bout following light transitions $\left(\boldsymbol{H}^{\prime}, \boldsymbol{N}^{\prime}\right)$ compared with $h d c+/-$ (cyan) and $h d c-/-$ (blue) siblings. There is no significant difference between $T g(h s p: h c r t /+) ; ~ h d c-/-$ and Tg(hsp:hcrt/ +); $h d c+/-$ larvae, except that Tg(hsp:hcrt/+); $h d c-/-$ larvae have shorter sleep bouts at night than Tg(hsp:hcrt/+); $h d c+/-$ larvae 
continued

$\left(\boldsymbol{M}^{\prime}\right)$. Line and bar graphs represent the mean \pm SEM for four experiments combined; $n$ indicates the number of animals analyzed. $* p<0.05 ; * * p<0.01 ; * * * p<0.001$ for the indicated comparisons by two-way ANOVA with Tukey's HSD test.

predicted null mutation in the single zebrafish hypocretin receptor (hcrtr) ortholog exhibit fragmented sleep (Yokogawa et al., 2007), similar to mammals that lack Hcrt signaling (Chemelli et al., 1999; Lin et al., 1999; Peyron et al., 2000). Consistent with a previous study that failed to observe a behavioral phenotype in hcrtr-/- zebrafish larvae (Appelbaum et al., 2009), we observed no sleep/ wake phenotypes in hcrtr-/-; hdc+/- larvae compared with their hcrtr+/-; hdc+/- siblings (Fig. 16). We also did not observe differences between hcrtr-/-; hdc +/- larvae and their hcrtr-l-; hdc-l- siblings (Fig. 16). These results suggest that the absence of sleep/wake phenotypes in hdc and hcrtr mutant zebrafish larvae is not due to functional redundancy of these two arousal-promoting systems.

Narcoleptic subjects are reported to have an increased number of histaminergic neurons in the TMN compared with controls (John et al., 2013; Valko et al., 2013), whereas histamine levels are reduced in animal and human forms of narcolepsy and idiopathic hypersomnia (Nishino et al., 2001; Kanbayashi et al., 2009). In contrast to studies reporting increased histaminergic cells in narcoleptics and similar to one study of Hcrt-deficient mice (John et al., 2013), we found no difference in the number of hdc-expressing neurons in hcrtr $+/+, h c r t r+/-$, and hcrtr-/- sibling zebrafish larvae (Fig. 17A). It is possible that effects on the histaminergic system requires years of reduced Hcrt signaling, which is visible in human patients at autopsy but not in rodents sacrificed at 4-10 months or in zebrafish larvae. Alternatively, effects on histaminergic cells in human narcolepsy may result from autoimmune effects of the disease that are absent in animal models (Shan et al., 2015).

We also tested whether the absence of histamine signaling affects the number of larval zebrafish hortexpressing neurons, but we observed no difference among $h d c+/+$, $h d c+/-$, and $h d c-/-$ siblings (Fig. 17B). This result contrasts with a previous report in which zebrafish larvae treated with an $h d c$-specific morpholino or the Hdc inhibitor $\alpha$-FMH were found to have fewer hortexpressing neurons (Sundvik et al., 2011). The basis for this discrepancy is unclear, but it might result from offtarget effects of the morpholino and $\alpha-\mathrm{FMH}$, or compensatory effects in the constitutive hdc mutant. We conclude that loss of histamine does not affect the specification of hcrt-expressing neurons, and loss of Hcrt signaling does not affect the specification of $h d c$-expressing neurons, in zebrafish larvae.

\section{Discussion}

Histamine is thought to be a wake-promoting neuromodulator primarily, because administration of histamine and pharmacological inhibition of histamine degradation increases wakefulness (Lin et al., 1988; Ramesh et al., 2004), whereas pharmacological inhibition of histamine synthesis or Hrh1 signaling increases sleep (Kiyono et al.,
1985; Lin et al., 1988). However, genetic studies have shown weak and often conflicting effects. For example, hdc mutant mice have normal sleep/wake amounts over a 24-h period (Parmentier et al., 2002) and only exhibit an arousal defect when high vigilance is required (Parmentier et al., 2002; Anaclet et al., 2009). Similarly, hrh1 mutant mice have normal sleep/wake amounts over a 24-h period, with increased locomotor activity during the light period and slightly less activity during the dark period (Inoue et al., 1996; Huang et al., 2006).

Study of the role of histamine in mammalian sleep is complicated by its production in basophils and mast cells, where it signals via Hrh1 and Hrh2 to affect immune system function (Steinman, 2004; Haas et al., 2008; Jutel et al., 2009). Hrh1 and Hrh2 signaling can also be activated by stress via the corticotrophin-releasing hormonemast cell-histamine axis (Elenkov et al., 2005; Tsatsoulis, 2009). The immune system has profound effects on sleep (Steinman, 2004; Opp, 2005; Rihel et al., 2010) and could account for histamine-dependent sleep phenotypes in mammals (Bollinger et al., 2009; Chikahisa et al., 2013). Indeed, inducing release of histamine from mast cells enhances wakefulness in WT mice, and the sleepinducing effect of Hrh1 antagonists is suppressed in mast cell-deficient mice (Chikahisa et al., 2013). These results suggest that histamine produced by cells of the immune system plays an important role in arousal. In contrast, histamine is exclusively produced in the TMN in zebrafish (Da'as et al., 2011), and thus is unlikely to be involved in regulating immune system function, although this remains a possibility. In Drosophila, histamine is primarily produced in photoreceptors, and perturbation of histamine signaling affects the detection of light cues that regulate sleep (Melzig et al., 1996; Nässel, 1999). Thus, while a Drosophila hdc hypomorph exhibits increased daytime sleep (Oh et al., 2013), this may result from impaired vision.

A previous zebrafish study used a morpholino antisense oligonucleotide to knock down Hdc levels and found that these animals were less active during the day and failed to respond to a lights off stimulus (Sundvik et al., 2011). The discrepancy between our genetic results and the morpholino data could be due to morpholino-induced nonspecific toxicity that can affect behavior (Bedell et al., 2011). Indeed, $80 \%$ of morpholino-induced phenotypes are not reproduced in mutants (Kok et al., 2015), suggesting that many of these phenotypes are artifacts, although some phenotypes may be masked in mutants but not in morphants due to a poorly understood compensatory mechanism (Rossi et al., 2015). Small molecule inhibition of Hrh1 has also been reported to result in reduced locomotor activity in zebrafish larvae (Peitsaro et al., 2007; Renier et al., 2007; Rihel et al., 2010; Sundvik et al., 2011), but drugs can have off-target effects and lack the specificity of genetic perturbations. To avoid these problems, we 
A

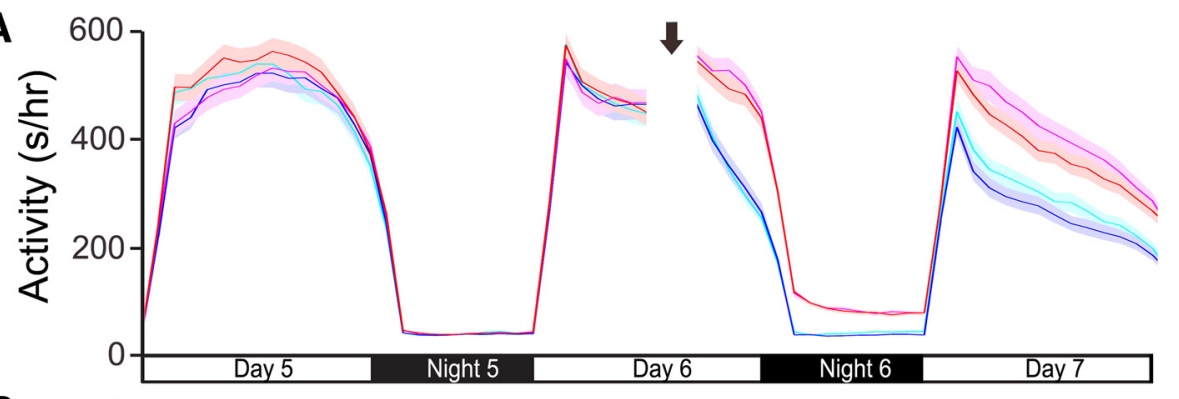

B

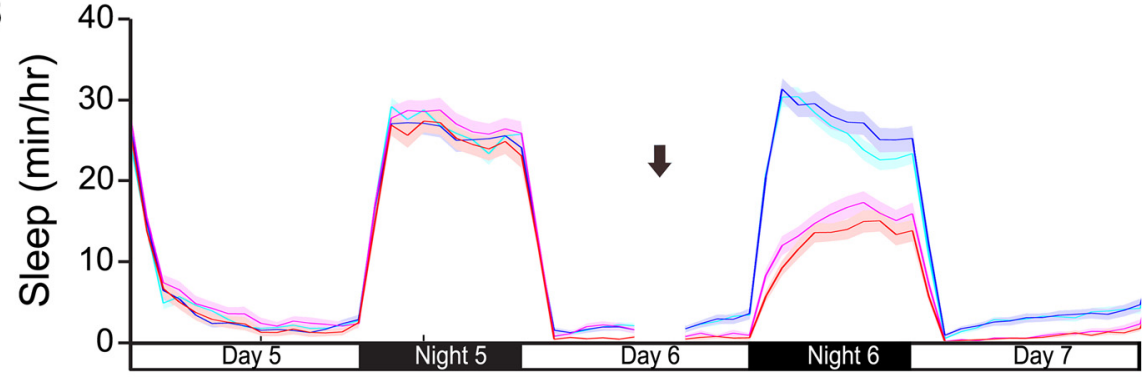

- hrh1+/- $\quad n=83$

- hrh1-/- $\quad n=70$

- hsp:hort/+; hrh1+/- $n=68$

— hsp:hcrt/+; hrh1-/- $\quad n=72$
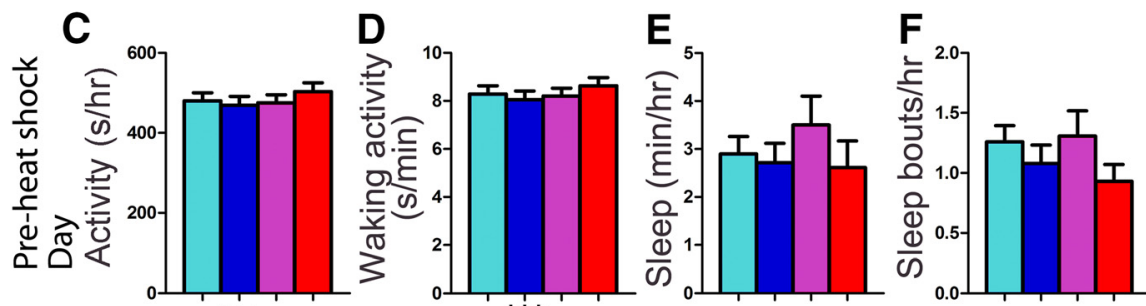

G
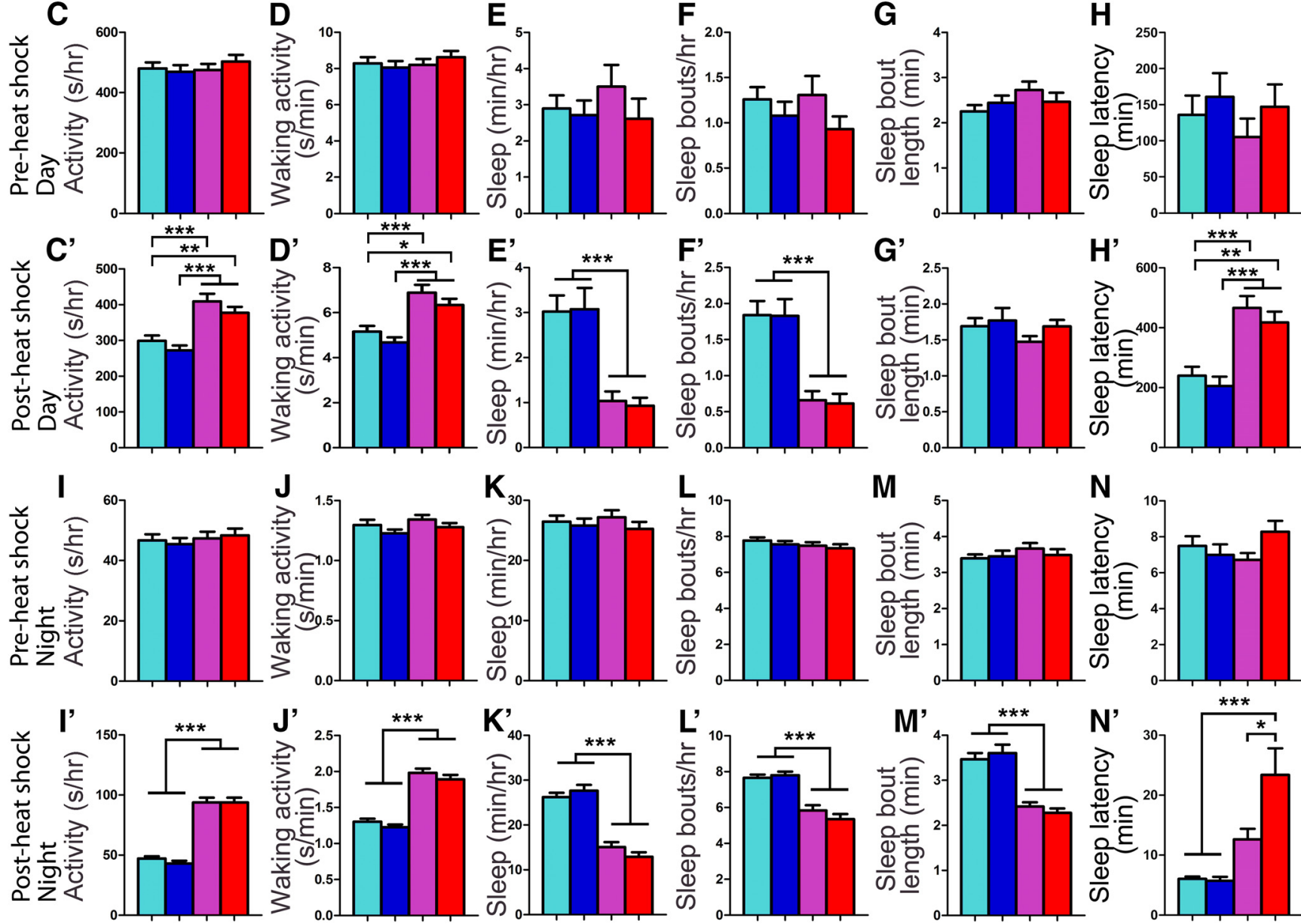

Figure 14. Hypocretin overexpression-induced hyperactivity and reduced sleep does not require hrh1. Before heat shock-induced Hcrt overexpression, there is no difference in all measured sleep/wake parameters between animals of each genotype $(\boldsymbol{A}-\boldsymbol{H}, \boldsymbol{I}-\boldsymbol{N})$. Following a 1-h heat shock during the afternoon of day 6 (indicated by arrows in line graphs), during both the day and night, both Tg(hsp:hcrt/+); $h r h 1-/-\left(\right.$ red) and Tg(hsp:hcrt/+); $h r h 1+/-$ (magenta) larvae exhibit more activity $\left(\boldsymbol{A}, \boldsymbol{C}^{\prime}, \boldsymbol{I}^{\prime}\right)$ and waking activity ( $\boldsymbol{D}^{\prime}$, $\left.\boldsymbol{J}^{\prime}\right)$, less sleep $\left(\boldsymbol{B}, \boldsymbol{E}^{\prime}, \boldsymbol{K}^{\prime}\right)$, fewer sleep bouts $\left(\boldsymbol{F}^{\prime}, \boldsymbol{L}^{\prime}\right)$, and increased latency to first sleep bout following light transitions $\left(\boldsymbol{H}^{\prime}, \boldsymbol{N}^{\prime}\right)$ compared with $h r h 1+/-$ (cyan) and $h r h 1-/-$ (blue) siblings. There is no significant difference between $T g(h s p: h c r t /+) ; h r h 1-/-$ and Tg(hsp: $h c r t /+) ; h r h 1+/-$ larvae after heat shock, except that Tg(hsp:hcrt/+); $h r h 1-/-$ larvae have a longer latency to first sleep bout at night 
continued

than their Tg(hsp:hcrt/+); $h r h 1+/-$ siblings $\left(\mathbf{N}^{\prime}\right)$. Line and bar graphs represent the mean \pm SEM for four experiments combined; $n$ indicates the number of animals analyzed. $* p<0.05 ; * * p<0.01 ; * * * p<0.001$ for the indicated comparisons by two-way ANOVA with Tukey's HSD test.

generated zebrafish that contain predicted null mutations in hdc and in histamine receptors, the first such study in a diurnal vertebrate animal. There are two important caveats associated with this approach. First, while we show that hdc mutant zebrafish produce little or no histamine, we only have molecular evidence based on genome sequence that the histamine receptor mutations generate nonfunctional proteins. It would be preferable to show loss of histamine receptor protein using Western blotting or IHC, but antibodies specific for the zebrafish histamine receptors are not available. It is possible for a point mutation that generates a premature stop codon to be bypassed due to read-through translation (Dunn et al., 2013), and thus the zebrafish hrh1 mutant might retain some function. However, the other zebrafish histamine receptor mutants reported in this study contain an insertion or deletion that causes a shift in translational reading frame, and thus abnormal protein sequence and several premature stop codons, before domains that are essential for protein function, including multiple transmembrane domains. It is therefore unlikely that these mutants retain any function. It would also be surprising for any of the histamine receptor mutants to have sleep/wake phenotypes given that hdc mutant zebrafish, which we show have little or no histamine, lack such phenotypes. Second, histamine receptors were annotated based on reciprocal BLAST searches among the human, mouse, and zebrafish genomes, which revealed a high degree of amino acid sequence conservation, and allowed unambiguous annotation of specific receptors. However, we cannot rule out the possibility that additional functionally redundant histamine receptors are present in the zebrafish genome, which could explain the absence of robust histamine receptor mutant phenotypes. Indeed, this may also be the case for rodents, which could explain why rodent histamine receptor mutants exhibit sleep/wake phenotypes that are much weaker than those observed using drugs that affect histamine signaling.

Similar to the rodent hdc mutant (Parmentier et al., 2002), we found that hdc mutant zebrafish larvae have largely normal sleep/wake behaviors. We also failed to detect major sleep/wake defects in larval zebrafish containing mutations in histamine receptors, which is generally consistent with mouse genetic studies. The murine hrh1 knock-out displays reduced exploratory behavior in new environments but normal total locomotor activity over a 24-h period, with less slightly less activity at night but more activity during the day (Inoue et al., 1996; Yanai et al., 1998; Huang et al., 2006). We found that hrh1 mutant zebrafish lack major defects in sleep/wake behaviors, although they are hyperactive at night, similar to hrh1-/- mice, which are hyperactive during the day, the rest period of these nocturnal animals (Inoue et al., 1996). Despite the lack of robust sleep phenotypes in rodent and zebrafish hdc and hrh1 mutants, we do not suggest that histamine signaling has no role in arousal. Indeed, several studies have shown that acute pharmacological manipulation of histamine signaling has profound effects on arousal in zebrafish (Peitsaro et al., 2007; Renier et al., 2007; Rihel et al., 2010; Sundvik et al., 2011) and mammals (Thakkar, 2011). The most interesting potential implication of this discrepancy is that there are mechanisms that compensate for the constitutive loss of histamine signaling on arousal, possibly by strengthening of other arousal systems, which does not immediately occur in response to acute pharmacological manipulations. Consistent with this possibility, the sedating effect of the Hrh1 antagonist diphenhydramine becomes indistinguishable from placebo after only four days of treatment in humans (Richardson et al., 2002). Similar discrepancies between strong effects of acute pharmacological manipulation compared with subtle phenotypes for constitutive genetic loss-of-function have also been shown in mammals for other sleep regulatory systems, including noradrenaline and adenosine (Berridge et al., 2012; Brown et al., 2012), suggesting that this compensation may be a general feature of sleep control. Alternatively, the sedating effects of Hrh1 antagonists may be due to off-target effects. Indeed, the Hrh1 antagonist pyrilamine has local anesthetic, anti$\alpha$-adrenergic, and antimuscarinic activity, and other functions at non-Hrh1 sites (Hill and Young, 1980; Roberts and Calcutt, 1983; Nicholson et al., 1985; Leurs et al., 1995). hrh2 is expressed sparsely and diffusely in the rodent brain, mediating inhibition in the suprachiasmatic nucleus and ventromedial hypothalamus and excitation in the dentate gyrus, hippocampus, thalamus, and cortex (Karlstedt et al., 2001; Haas et al., 2008). Studies of the effect of pharmacological inhibition of Hrh2 on sleep-wake cycles have yielded inconsistent findings. For example, the Hrh2 antagonists zolantidine and cimetidine have no effect on sleep in rats (Monti et al., 1986; Monti et al., 1990), whereas injection of the Hrh2 agonist impromidine in the preoptic region promotes wakefulness in cats. The Hrh2 antagonist ranitidine, which is used to treat stomach acid overproduction, increases feline sleep (Lin et al., 1994; Lin et al., 1996). It is unclear whether this is a direct effect of Hrh2 inhibition, or indirectly due to the alleviation of peripheral symptoms that disrupt sleep. hrh2 knock-out mice are hypoactive (Dai et al., 2007), but sleep studies have not been described for these animals. Here, we show that hrh2 mutant zebrafish exhibit normal sleep/ wake behaviors. Thus, although Hrh2 may be involved in sleep regulation in the cat, there is no genetic evidence that Hrh2 regulates sleep in zebrafish or rodents.

Hrh3 acts as an autoreceptor to control histamine synthesis, release, and activity of histaminergic neurons (Morisset et al., 2000; Arrang et al., 2007) and as a heteroreceptor to influence the release of other neurotransmitters (Schlicker et al., 1994; Samaranayake et al., 2016). Mammalian hrh3 is expressed in the TMN as well as in 
A
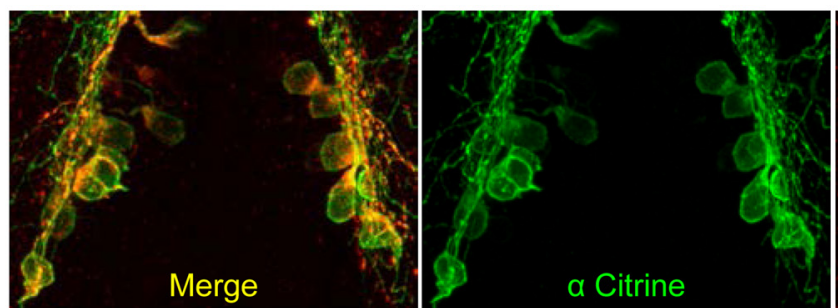

B

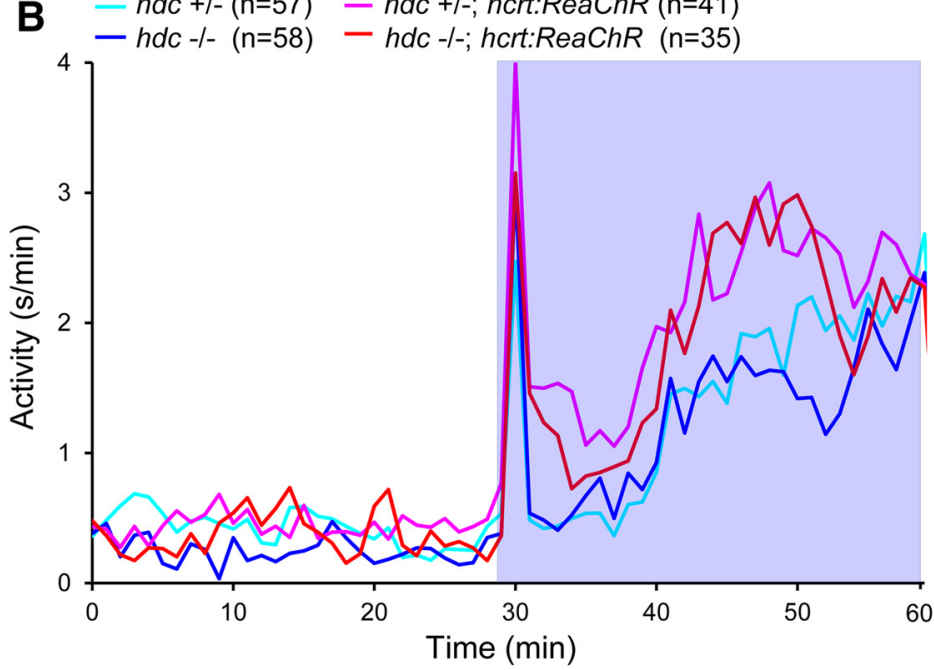

D $\quad-h r h 1+/-(\mathrm{n}=94) \quad-h r h 1+/-; h c r t: R e a C h R(\mathrm{n}=102)$

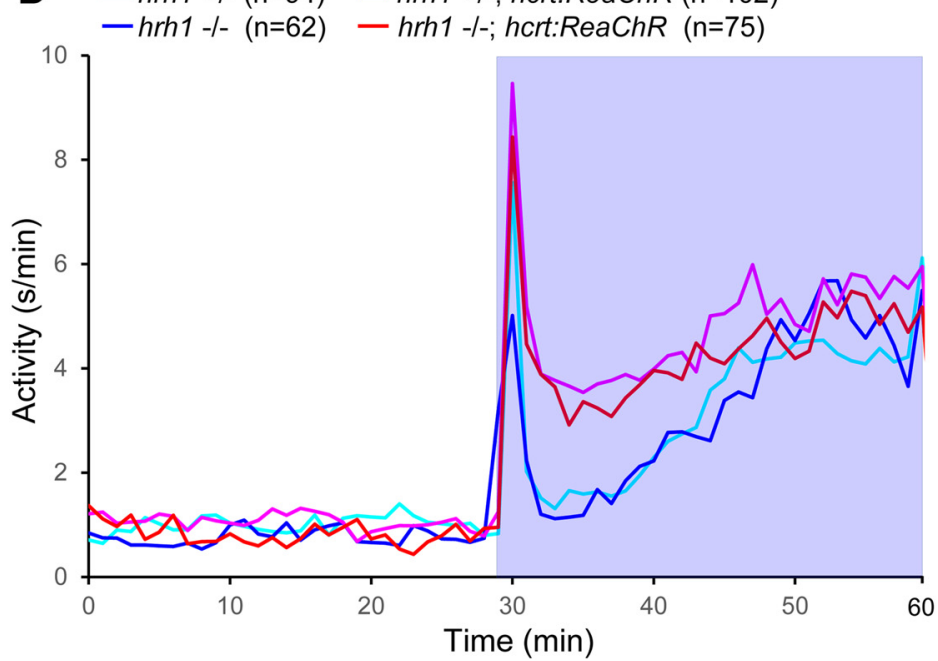

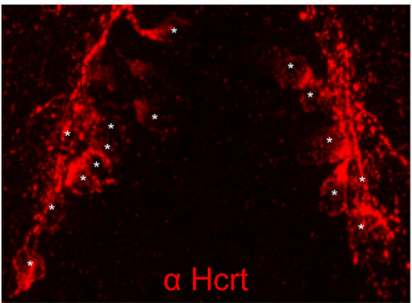

C

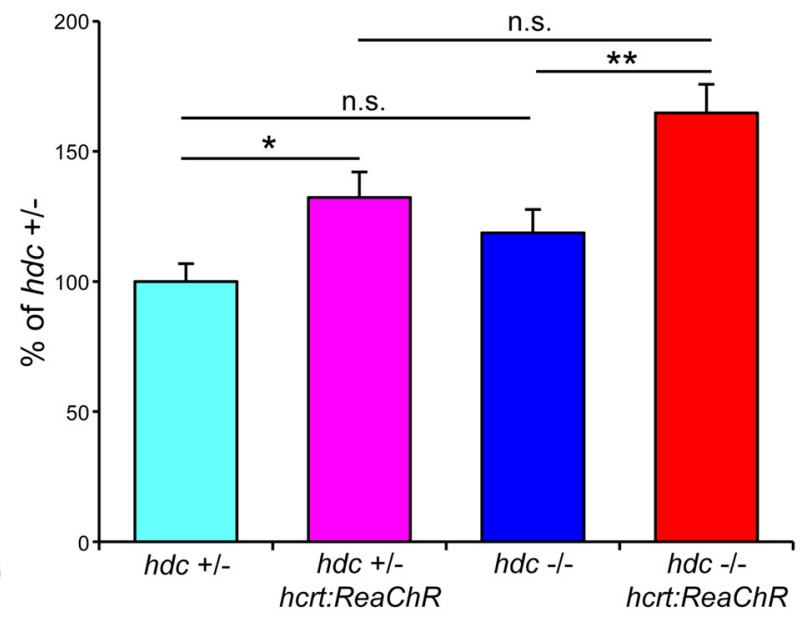

E

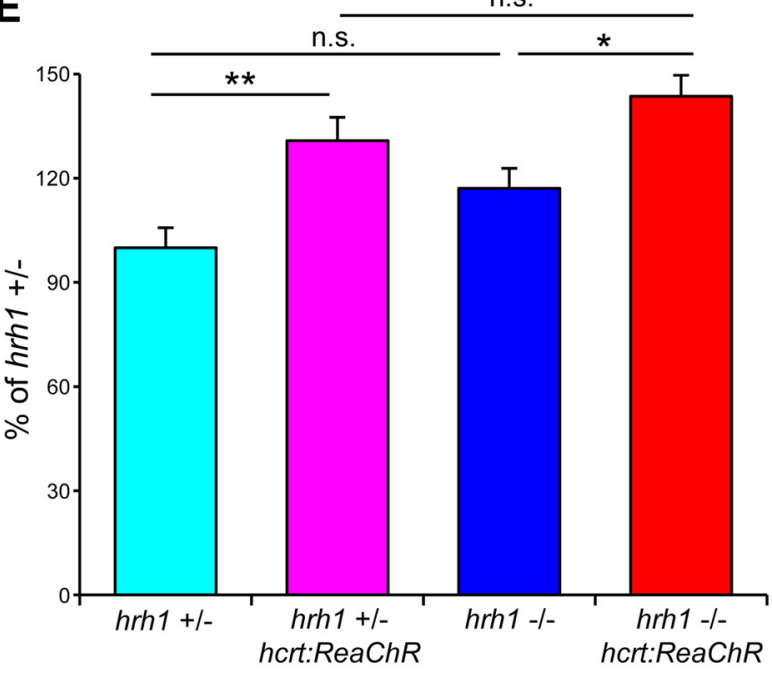

Figure 15. Optogenetic stimulation of hcrt-expressing neurons promotes locomotor activity in $h d c$ and $h r h 1$ mutant larvae. $\boldsymbol{A}$, ReaChR-mCitrine is specifically expressed in hort-expressing neurons in Tg(hcrt:ReaChR-mCitrine) larvae. IHC using antibodies specific for mCitrine (green) and Hcrt (red) are shown. Asterisks indicate the soma of neurons labeled with the Hcrt-specific antibody. A maximum intensity projection of a ventral view of a $5 \mathrm{dpf}$ larval brain is shown. Scale bar: $20 \mu \mathrm{m}$. $\boldsymbol{B}, \boldsymbol{D}$, Locomotor activity before (white background) and during (blue background) blue light stimulation. $\boldsymbol{C}, \boldsymbol{E}$, Locomotor activity during blue light stimulation normalized to average prestimulation activity level for each genotype and expressed relative to ReaChR negative siblings for $h d c+/-$ $(\boldsymbol{C})$ and $h r h 1+/-(\boldsymbol{E})$ larvae. Data are pooled from three $(\boldsymbol{B}, \boldsymbol{C})$ or four $(\boldsymbol{D}, \boldsymbol{E})$ experiments and are represented as mean $\pm \mathrm{SEM}$; $n$ indicates the number of animals analyzed. $* p<0.05$; $* * p<0.01$; n.s. $=$ not significant $(p>0.05)$ by one-way ANOVA with Tukey's HSD test.

brain regions that receive histaminergic input (Haas et al., 2008). Hrh3 antagonists, such as ciproxifan and thioperamide, promote wake by suppressing cortical slow activity $(0.5-8 \mathrm{~Hz})$ and enhancing the frequency and amplitude of cortical fast rhythms $(30-60 \mathrm{~Hz})$, which is associated with higher cognitive functions (Toyota et al., 2002; Parmentier et al., 2007), and the Hrh3 antagonist pitolisant was found to reduce daytime sleepiness in $23-38 \%$ of self-reports 

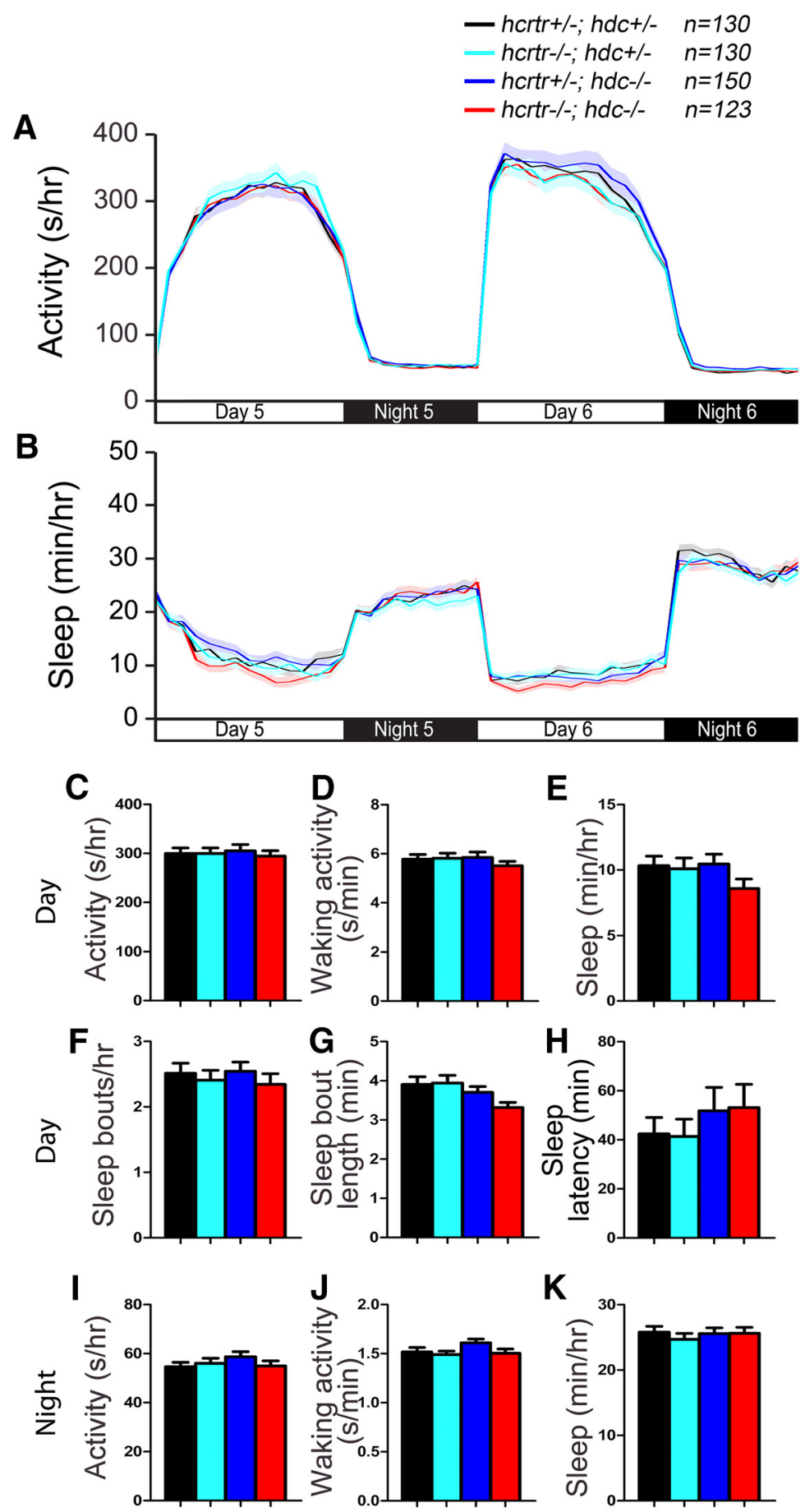

K
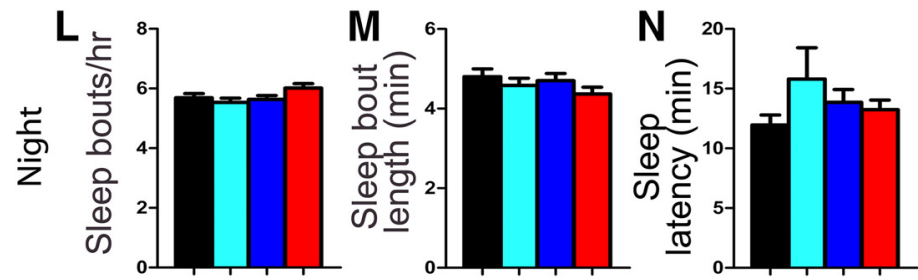

Figure 16. hcrtr; hdc double mutant larvae exhibit normal sleep/wake behaviors. hcrtr-/-; hdc-/- (red), hcrtr+/-; hdc+/- (black), hcrtr-l-; hdc+l- (cyan), and hcrtr+/-; hdc-/- (blue) larvae exhibit similar amounts of all measured sleep/wake parameters. Line and bar graphs represent the mean \pm SEM for six experiments combined. Bar graphs show total values for day and night periods; $n$ indicates the number of animals analyzed. $p>0.05$ for all comparisons by one-way ANOVA with Tukey's HSD test. 

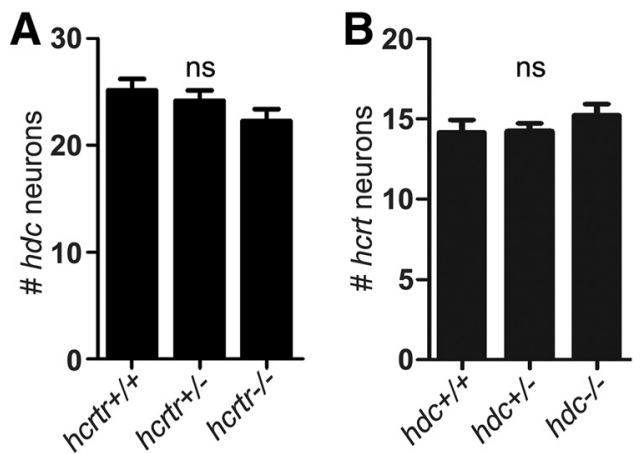

Figure 17. Absence of Hcrt or histamine signaling does not affect the number of histaminergic or Hcrt neurons. $\boldsymbol{A}$, There is no difference in the number of $h d c$-expressing neurons between $h c r t r+/+, h c r t r+/-$, and hcrtr-/- larvae $(n=21,42$, and 27, respectively). $\boldsymbol{B}$, There is no difference in the number of hcrtexpressing neurons between $h d c+/+,+/-$, and $h d c-/-$ siblings ( $n=13,17$, and 13, respectively). $h d c$ and $h c r t r$ expression was assayed by ISH at $5 \mathrm{dpf}$. ns = no statistically significant difference $(p>0.05)$ by one-way ANOVA with Tukey's HSD test.

from patients with hypersomnia (Leu-Semenescu et al., 2014). However, hrh3 knock-out mice are hypoactive (Toyota et al., 2002; Gondard et al., 2013) and only exhibit increased wake duration during motor challenge (Gondard et al., 2013). We found that $h r h 3$ mutant zebrafish larvae have essentially normal sleep/wake behaviors, with slightly increased sleep bout length during the day and night. This subtle phenotype contrasts with the hrh3 knock-out mouse, which exhibits sleep fragmentation at night (Gondard et al., 2013).

Together, our genetic studies suggest that constitutive lack of histamine signaling has minimal effects on sleep/ wake behaviors in zebrafish, similar to results obtained using genetics in mice. However, mouse studies identified subtle mutant phenotypes, particularly arousal defects when high vigilance is required, such as on introduction to a novel environment, in response to changes in lighting, or motor challenge to reach palatable food (Inoue et al., 1996; Kubota et al., 2002; Parmentier et al., 2002; Abe et al., 2004; Anaclet et al., 2009; Thakkar, 2011; Gondard et al., 2013). To test whether environmental challenges might uncover behavioral defects in zebrafish histamine mutants, we subjected hdc and $h r h 1$ mutants to a mechano-acoustic stimulus and to 1-h periods of alternating light and darkness, both of which induce arousal in zebrafish larvae. These assays failed to reveal behavioral defects in either mutant, although it remains possible that other environmental perturbations or more sensitive assays might detect subtle phenotypes. Alternatively, histamine may be required to regulate arousal in adult animals and not during earlier developmental stages. This question can be addressed by studying histamine mutants in adult zebrafish and in juvenile rodents. Experiments using conditional mutants may also determine whether the essentially normal sleep/wake behaviors observed in mutants is due to compensatory mechanisms during development. It is also possible that additional proteins can act as histamine receptors but have not been targeted in our zebrafish study or in previous mouse studies. Finally, it is possible that the strong effects of acute pharmacological manipulation of histamine signaling are due to off-target effects that are independent of histamine signaling.

Genetic and optogenetic studies in rodents (Chemelli et al., 1999; Adamantidis et al., 2007) and zebrafish (Prober et al., 2006; Singh et al., 2015; Chen et al., 2016) have shown that Hcrt peptides and hcrt-expressing neurons play key roles in promoting arousal. Several lines of evidence suggest that the Hcrt system promotes arousal via histamine signaling in rodents. First, Hcrt neurons project to histaminergic neurons in the TMN, which express Hcrt receptors 1 and 2 (Bayer et al., 2001; Eriksson et al., 2001). Second, perfusion of Hcrt peptide into the TMN increases histamine release (Huang et al., 2001; Ishizuka et al., 2002), bath application of Hcrt excites TMN neurons in hypothalamic slices (Eriksson et al., 2001), and optogenetic stimulation of hcrt-expressing neurons elicits fast postsynaptic currents in histaminergic neurons (Eriksson et al., 2001; Schöne et al., 2012), suggesting that Hcrt and hort-expressing neurons can directly stimulate TMN histaminergic neurons. Third, arousal induced by Hcrt infusion is blocked in hrh1 knock-out mice (Huang et al., 2001) and by the Hrh1 antagonist pyrilamine in rats (Yamanaka et al., 2002; Shigemoto et al., 2004), suggesting that Hrh1 signaling is required for Hcrt-induced arousal. In contrast, we found that mutation of either hdc or hrh1 does not block arousal induced by Hcrt overexpression or stimulation of hcrt-expressing neurons in zebrafish larvae. A possible explanation for the discrepancy between larval zebrafish and adult rodents is that histamine signaling is only required for Hcrt-induced arousal in adult animals. Consistent with this possibility, hert-expressing neurons do not project to the TMN in zebrafish larvae (Prober et al., 2006), but they do so in adults (Kaslin et al., 2004). Alternatively, the requirement of Hrh1 for Hcrt-induced arousal in rodents may be due to Hrh1-mediated signaling in the immune system that is absent in zebrafish. The discrepancy between rodents and zebrafish may also reflect species-specific differences in mechanisms of Hcrt-induced arousal. However, our results are consistent with the findings that Hrh1 is not required for endogenous Hcrt signaling in rodents (Hondo et al., 2010) and that stimulation of hcrt-expressing neurons increases the probability of an awakening event in both WT and hdc mutant mice to similar extents (Carter et al., 2009). In rodents, Hcrt likely also promotes wakefulness through other arousal centers, including the noradrenergic locus coeruleus, the serotonergic dorsal raphe, and cholinergic neurons in the basal forebrain (Date et al., 1999; Bourgin et al., 2000; Eggermann et al., 2001; Liu et al., 2002; Fadel and Burk, 2010). We found that impaired noradrenergic signaling partially blocks arousal induced by Hcrt overexpression and stimulation of hcrt-expressing neurons in zebrafish (Singh et al., 2015), suggesting that both noradrenergic and additional arousal centers mediate Hcrt-induced arousal in zebrafish.

In summary, our genetic data suggest that histamine and its receptors are not required for normal sleep/wake behaviors in a diurnal vertebrate animal whose histamine production is limited to the brain. Our results are similar to those obtained using genetics in mice (Inoue et al., 1996; Yanai et al., 1998; Parmentier et al., 2002; Abe et al., 2004; Anaclet 
et al., 2009), which found only subtle sleep/wake phenotypes, and contrast with acute pharmacological manipulations of histamine synthesis and signaling, which results in strong effects on arousal in both mammals (Kiyono et al., 1985; Lin et al., 1988; Ramesh et al., 2004) and zebrafish (Peitsaro et al., 2007; Renier et al., 2007; Rihel et al., 2010; Sundvik et al., 2011). These results suggest either that mechanisms compensate for constitutive loss of histamine signaling in mutants but not in response to acute pharmacological manipulations, or that phenotypes observed using pharmacology are due to off-target effects. While it remains unclear which possibility is correct, these studies suggest that histamine signaling plays similar roles in regulating zebrafish and mammalian sleep/wake states.

\section{References}

Abe H, Honma S, Ohtsu H, Honma K (2004) Circadian rhythms in behavior and clock gene expressions in the brain of mice lacking histidine decarboxylase. Mol Brain Res 124:178-187. CrossRef Medline

Adamantidis AR, Zhang F, Aravanis AM, Deisseroth K, de Lecea L (2007) Neural substrates of awakening probed with optogenetic control of hypocretin neurons. Nature 450:420-424. CrossRef Medline

Airaksinen MS, Paetau A, Paljärvi L, Reinikainen K, Riekkinen P, Suomalainen R, Panula P (1991) Histamine neurons in human hypothalamus: anatomy in normal and Alzheimer diseased brains. Neuroscience 44:465-481. Medline

Allada R, Siegel JM (2008) Unearthing the phylogenetic roots of sleep. Curr Biol 18:R670-R679. CrossRef Medline

Anaclet C, Parmentier R, Ouk K, Guidon G, Buda C, Sastre JP, Akaoka $\mathrm{H}$, Sergeeva OA, Yanagisawa M, Ohtsu H, Franco P, Haas $\mathrm{HL}$, Lin JS (2009) Orexin/hypocretin and histamine: distinct roles in the control of wakefulness demonstrated using knock-out mouse models. J Neurosci 29:14423-14438. CrossRef Medline

Appelbaum L, Wang GX, Maro GS, Mori R, Tovin A, Marin W, Yokogawa T, Kawakami K, Smith SJ, Gothilf Y, Mignot E, Mourrain $P(2009)$ Sleep-wake regulation and hypocretin-melatonin interaction in zebrafish. Proc Natl Acad Sci USA 106:21942-21947. CrossRef Medline

Arrang JM, Morisset S, Gbahou F (2007) Constitutive activity of the histamine H3 receptor. Trends Pharmacol Sci 28:350-357. CrossRef Medline

Asakawa K, Kawakami K (2009) The Tol2-mediated Gal4-UAS method for gene and enhancer trapping in zebrafish. Methods 49:275-281. CrossRef Medline

Bayer L, Eggermann E, Serafin M, Saint-Mleux B, Machard D, Jones B, Mühlethaler M (2001) Orexins (hypocretins) directly excite tuberomammillary neurons. Eur J Neurosci 14:1571-1575. Medline

Bedell VM, Westcot SE, Ekker SC (2011) Lessons from morpholinobased screening in zebrafish. Brief Funct Genomics 10:181-188. CrossRef Medline

Berridge CW, Schmeichel BE, España RA (2012) Noradrenergic modulation of wakefulness/arousal. Sleep Med Rev 16:187-197. CrossRef Medline

Bollinger T, Bollinger A, Skrum L, Dimitrov S, Lange T, Solbach W (2009) Sleep-dependent activity of T cells and regulatory T cells. Clin Exp Immunol 155:231-238. CrossRef Medline

Bourgin P, Huitrón-Résendiz S, Spier AD, Fabre V, Morte B, Criado JR, Sutcliffe JG, Henriksen SJ, de Lecea L (2000) Hypocretin-1 modulates rapid eye movement sleep through activation of locus coeruleus neurons. J Neurosci 20:7760-7765. Medline

Brown RE, Basheer R, McKenna JT, Strecker RE, McCarley RW (2012) Control of sleep and wakefulness. Physiol Rev 92:10871187. CrossRef Medline

Burgess HA, Granato M (2007) Modulation of locomotor activity in larval zebrafish during light adaptation. J Exp Biol 210:2526-2539. CrossRef Medline
Campbell SS, Tobler I (1984) Animal sleep: a review of sleep duration across phylogeny. Neurosci Biobehav Rev 8:269-300. Medline

Carter ME, Adamantidis A, Ohtsu H, Deisseroth K, de Lecea L (2009) Sleep homeostasis modulates hypocretin-mediated sleep-towake transitions. J Neurosci 29:10939-10949. CrossRef Medline

Chemelli RM, Willie JT, Sinton CM, Elmquist JK, Scammell T, Lee C, Richardson JA, Williams SC, Xiong Y, Kisanuki Y, Fitch TE, Nakazato M, Hammer RE, Saper CB, Yanagisawa M (1999) Narcolepsy in orexin knockout mice: molecular genetics of sleep regulation. Cell 98:437-451. Medline

Chen S, Chiu CN, McArthur KL, Fetcho JR, Prober DA (2016) TRP channel mediated neuronal activation and ablation in freely behaving zebrafish. Nat Methods 13:147-150. CrossRef Medline

Chikahisa S, Kodama T, Soya A, Sagawa $Y$, Ishimaru $Y$, Sei $H$, Nishino S (2013) Histamine from brain resident MAST cells promotes wakefulness and modulates behavioral states. PLoS One 8:e78434. CrossRef Medline

Chiu CN, Prober DA (2013) Regulation of zebrafish sleep and arousal states: current and prospective approaches. Front Neural Circuits 7:58CrossRef Medline

Da'as S, Teh EM, Dobson JT, Nasrallah GK, McBride ER, Wang H, Neuberg DS, Marshall JS, Lin TJ, Berman JN (2011) Zebrafish mast cells possess an $\mathrm{F} c \varepsilon$ RI-like receptor and participate in innate and adaptive immune responses. Dev Comp Immunol 35:125134. CrossRef Medline

Dai H, Kaneko K, Kato H, Fujii S, Jing Y, Xu A, Sakurai E, Kato M, Okamura N, Kuramasu A, Yanai K (2007) Selective cognitive dysfunction in mice lacking histamine $\mathrm{H} 1$ and $\mathrm{H} 2$ receptors. Neurosci Res 57:306-313. CrossRef Medline

Date Y, Ueta Y, Yamashita H, Yamaguchi H, Matsukura S, Kangawa K, Sakurai T, Yanagisawa M, Nakazato M (1999) Orexins, orexigenic hypothalamic peptides, interact with autonomic, neuroendocrine and neuroregulatory systems. Proc Natl Acad Sci USA 96:748-753. Medline

de Esch IJ, Thurmond RL, Jongejan A, Leurs R (2005) The histamine $\mathrm{H} 4$ receptor as a new therapeutic target for inflammation. Trends Pharmacol Sci 26:462-469. CrossRef Medline

Dunn JG, Foo CK, Belletier NG, Gavis ER, Weissman JS (2013) Ribosome profiling reveals pervasive and regulated stop codon readthrough in Drosophila melanogaster. eLife 2:e01179. CrossRef Medline

Eggermann E, Serafin M, Bayer L, Machard D, Saint-Mleux B, Jones BE, Mühlethaler M (2001) Orexins/hypocretins excite basal forebrain cholinergic neurones. Neuroscience 108:177-181. Medline

Elbaz I, Yelin-Bekerman L, Nicenboim J, Vatine G, Appelbaum L (2012) Genetic ablation of hypocretin neurons alters behavioral state transitions in zebrafish. J Neurosci 32:12961-12972. CrossRef Medline

Elenkov IJ, lezzoni DG, Daly A, Harris AG, Chrousos GP (2005) Cytokine dysregulation, inflammation and well-being. Neuroimmunomodulation 12:255-269. CrossRef Medline

Ericson H, Watanabe T, Kohler C (1987) Morphological analysis of the tuberomammillary nucleus in the rat brain: delineation of subgroups with antibody against L-histidine decarboxylase as a marker. J Comp Neur 263:1-24. CrossRef

Eriksson KS, Peitsaro N, Karlstedt K, Kaslin J, Panula P (1998) Development of the histaminergic neurons and expression of histidine decarboxylase mRNA in the zebrafish brain in the absence of all peripheral histaminergic systems. Eur J Neurosci 10:3799-3812.

Eriksson KS, Sergeeva O, Brown RE, Haas HL (2001) Orexin/hypocretin excites the histaminergic neurons of the tuberomammillary nucleus. J Neurosci 21:9273-9279. Medline

Fadel J, Burk JA (2010) Orexin/hypocretin modulation of the basal forebrain cholinergic system: role in attention. Brain Res 1314: 112-123. CrossRef Medline

Faraco JH, Appelbaum L, Marin W, Gaus SE, Mourrain P, Mignot E (2006) Regulation of hypocretin (orexin) expression in embryonic zebrafish. J Biol Chem 281:29753-29761. CrossRef Medline

Gondard E, Anaclet C, Akaoka H, Guo RX, Zhang M, Buda C, Franco P, Kotani H, Lin JS (2013) Enhanced histaminergic neurotransmission 
and sleep-wake alterations, a study in histamine H3-receptor knock-out mice. Neuropsychopharmacology 38:1015-1031. CrossRef Medline

Haas HL, Sergeeva OA, Selbach O (2008) Histamine in the nervous system. Physiol Rev 88:1183-1241. CrossRef Medline

Hill SJ, Young JM (1980) Histamine H1-receptors in the brain of the guinea-pig and the rat: differences in ligand binding properties and regional distribution. Br J Pharmacol 68:687-696. Medline

Hondo M, Nagai K, Ohno K, Kisanuki Y, Willie JT, Watanabe T, Yanagisawa M, Sakurai T (2010) Histamine-1 receptor is not required as a downstream effector of orexin-2 receptor in maintenance of basal sleep/wake states. Acta Physiol (Oxf) 198:287-294. CrossRef Medline

Huang ZL, Mochizuki T, Qu WM, Hong ZY, Watanabe T, Urade Y, Hayaishi O (2006) Altered sleep-wake characteristics and lack of arousal response to $\mathrm{H} 3$ receptor antagonist in histamine $\mathrm{H} 1$ receptor knockout mice. Proc Natl Acad Sci USA 103:4687-4692. CrossRef Medline

Huang ZL, Qu WM, Li WD, Mochizuki T, Eguchi N, Watanabe T, Urade Y, Hayaishi O (2001) Arousal effect of orexin A depends on activation of the histaminergic system. Proc Natl Acad Sci USA 98:9965-9970. CrossRef Medline

Inoue I, Yanai K, Kitamura D, Taniuchi I, Kobayashi T, Niimura K, Watanabe T, Watanabe T (1996) Impaired locomotor activity and exploratory behavior in mice lacking histamine $\mathrm{H} 1$ receptors. Proc Natl Acad Sci USA 93:13316-13320. Medline

Ishizuka T, Yamamoto Y, Yamatodani A (2002) The effect of orexin-A and $-\mathrm{B}$ on the histamine release in the anterior hypothalamus in rats. Neurosci Lett 323:93-96. Medline

Isken O, Maquat LE (2007) Quality control of eukaryotic mRNA: safeguarding cells from abnormal mRNA function. Genes Dev 21:1833-1856. CrossRef Medline

John J, Thannickal TC, McGregor R, Ramanathan L, Ohtsu H, Nishino S, Sakai N, Yamanaka A, Stone C, Cornford M, Siegel JM (2013) Greatly increased numbers of histamine cells in human narcolepsy with cataplexy. Ann Neurol 74:786-793. CrossRef Medline

Jutel M, Akdis M, Akdis CA (2009) Histamine, histamine receptors and their role in immune pathology. Clin Exp Allergy 39:17861800. CrossRef

Kanbayashi T, Kodama T, Kondo H, Satoh S, Inoue Y, Chiba S, Shimizu T, Nishino S (2009) CSF histamine contents in narcolepsy, idiopathic hypersomnia and obstructive sleep apnea syndrome. Sleep 32:181-187. Medline

Karlstedt K, Senkas A, Ahman M, Panula P (2001) Regional expression of the histamine $\mathrm{H}(2)$ receptor in adult and developing rat brain. Neuroscience 102:201-208. Medline

Kaslin J, Nystedt JM, Ostergård M, Peitsaro N, Panula P (2004) The orexin/hypocretin system in zebrafish is connected to the aminergic and cholinergic systems. J Neurosci 24:2678-2689. CrossRef Medline

Kettleborough RN, Busch-Nentwich EM, Harvey SA, Dooley CM, de Bruijn E, van Eeden F, Sealy I, White RJ, Herd C, Nijman IJ, Fényes F, Mehroke S, Scahill C, Gibbons R, Wali N, Carruthers S, Hall A, Yen J, Cuppen E, Stemple DL (2013) A systematic genome-wide analysis of zebrafish protein-coding gene function. Nature 496: 494-497. CrossRef Medline

Kiyono S, Seo ML, Shibagaki M, Watanabe T, Maeyama K, Wada H (1985) Effects of alpha-fluoromethylhistidine on sleep-waking parameters in rats. Physiol Behav 34:615-617. Medline

Kok FO, Shin M, Ni CW, Gupta A, Grosse AS, van Impel A, Kirchmaier BC, Peterson-Maduro J, Kourkoulis G, Male I, DeSantis DF, Sheppard-Tindell S, Ebarasi L, Betsholtz C, Schulte-Merker S, Wolfe SA, Lawson ND (2015) Reverse genetic screening reveals poor correlation between morpholino-induced and mutant phenotypes in zebrafish. Dev Cell 32:97-108. CrossRef Medline

Komori H, Nitta Y, Ueno H, Higuchi Y (2012) Structural study reveals that Ser-354 determines substrate specificity on human histidine decarboxylase. J Biol Chem 287:29175-29183.

Kubota Y, Ito C, Sakurai E, Sakurai E, Watanabe T, Ohtsu H (2002) Increased methamphetamine-induced locomotor activity and be- havioral sensitization in histamine-deficient mice. J Neurochem 83:837-845. Medline

Leu-Semenescu S, Nittur N, Golmard JL, Arnulf I (2014) Effects of pitolisant, a histamine $\mathrm{H} 3$ inverse agonist, in drug-resistant idiopathic and symptomatic hypersomnia: a chart review. Sleep Med 15:681-687. CrossRef Medline

Leurs R, Smit MJ, Timmerman H (1995) Molecular pharmacological aspects of histamine receptors. Pharmacol Ther 66:413-463. Medline

Lin JS, Dauvilliers Y, Arnulf I, Bastuji H, Anaclet C, Parmentier R, Kocher L, Yanagisawa M, Lehert P, Ligneau X, Perrin D, Robert P, Roux M, Lecomte JM, Schwartz JC (2008) An inverse agonist of the histamine $\mathrm{H}(3)$ receptor improves wakefulness in narcolepsy: studies in orexin-/- mice and patients. Neurobiol Dis 30:74-83. CrossRef Medline

Lin JS, Hou Y, Sakai K, Jouvet M (1996) Histaminergic descending inputs to the mesopontine tegmentum and their role in the control of cortical activation and wakefulness in the cat. J Neurosci 16: 1523-1537. Medline

Lin JS, Sakai K, Jouvet M (1988) Evidence for histaminergic arousal mechanisms in the hypothalamus of cat. Neuropharmacology 27: 111-122. Medline

Lin JS, Sakai K, Jouvet M (1994) Hypothalamo-preoptic histaminergic projections in sleep-wake control in the cat. Eur $\mathrm{J}$ Neurosci 6:618-625. Medline

Lin JY, Knutsen PM, Muller A, Kleinfeld D, Tsien RY (2013) ReaChR: a red-shifted variant of channelrhodopsin enables deep transcranial optogenetic excitation. Nat Neurosci 16:1499-1508. CrossRef Medline

Lin L, Faraco J, Li R, Kadotani H, Rogers W, Lin X, Qiu X, de Jong PJ, Nishino S, Mignot E (1999) The sleep disorder canine narcolepsy is caused by a mutation in the hypocretin (orexin) receptor 2 gene. Cell 98:365-376. Medline

Liu RJ, van den Pol AN, Aghajanian GK (2002) Hypocretins (orexins) regulate serotonin neurons in the dorsal raphe nucleus by excitatory direct and inhibitory indirect actions. J Neurosci 22:94539464. Medline

Martinez-Mir MI, Pollard H, Moreau J, Arrang JM, Ruat M, Traiffort E, Schwartz JC, Palacios JM (1990) Three histamine receptors $(\mathrm{H} 1$, $\mathrm{H} 2$ and $\mathrm{H} 3$ ) visualized in the brain of human and non-human primates. Brain Res 526:322-327. Medline

Melzig J, Buchner S, Wiebel F, Wolf R, Burg M, Pak WL, Buchner E (1996) Genetic depletion of histamine from the nervous system of Drosophila eliminates specific visual and mechanosensory behavior. J Comp Physiol A 179:763-773. Medline

Monti JM, Orellana C, Boussard M, Jantos H, Olivera S (1990) Sleep variables are unaltered by zolantidine in rats: are histamine $\mathrm{H} 2-$ receptors not involved in sleep regulation?. Brain Res Bull 25:229 231. Medline

Monti JM, Pellejero T, Jantos H (1986) Effects of H1- and H2histamine receptor agonists and antagonists on sleep and wakefulness in the rat. J Neural Transm 66:11. Medline

Morisset S, Rouleau A, Ligneau X, Gbahou F, Tardivel-Lacombe J, Stark H, Schunack W, Ganellin CR, Schwartz JC, Arrang JM (2000) High constitutive activity of native $\mathrm{H} 3$ receptors regulates histamine neurons in brain. Nature 408:860-864. CrossRef Medline

Nässel DR (1999) Histamine in the brain of insects: a review. Microsc Res Tech 44:121-136. CrossRef

Nicholson AN, Pascoe PA, Stone BM (1985) Histaminergic systems and sleep. Studies in man with $\mathrm{H} 1$ and $\mathrm{H} 2$ antagonists. Neuropharmacology 24:245-250. Medline

Nishino S, Fujiki N, Ripley B, Sakurai E, Kato M, Watanabe T, Mignot E, Yanai K (2001) Decreased brain histamine content in hypocretin/ orexin receptor-2 mutated narcoleptic dogs. Neurosci Lett 313: 125-128. Medline

Oh Y, Jang D, Sonn JY, Choe J (2013) Histamine-HisCl1 receptor axis regulates wake-promoting signals in Drosophila melanogaster. PLoS One 8:e68269. CrossRef Medline

Opp MR (2005) Cytokines and sleep. Sleep Med Rev 9:355-364. CrossRef Medline 
Panula P, Chazot PL, Cowart M, Gutzmer R, Leurs R, Liu WL, Stark $\mathrm{H}$, Thurmond RL, Haas HL (2015) International union of basic and clinical pharmacology. XCVIII. Histamine receptors. Pharmacol Rev 67:601-655. CrossRef Medline

Parmentier R, Anaclet C, Guhennec C, Brousseau E, Bricout D, Giboulot T, Bozyczko-Coyne D, Spiegel K, Ohtsu H, Williams M, Lin JS (2007) The brain H3-receptor as a novel therapeutic target for vigilance and sleep-wake disorders. Biochem Pharmacol 73: 1157-1171. CrossRef Medline

Parmentier R, Ohtsu H, Djebbara-Hannas Z, Valatx JL, Watanabe T, Lin JS (2002) Anatomical, physiological, and pharmacological characteristics of histidine decarboxylase knock-out mice: evidence for the role of brain histamine in behavioral and sleep-wake control. J Neurosci 22:7695-7711. Medline

Peitsaro N, Sundvik M, Anichtchik OV, Kaslin J, Panula P (2007) Identification of zebrafish histamine $\mathrm{H} 1, \mathrm{H} 2$ and $\mathrm{H} 3$ receptors and effects of histaminergic ligands on behavior. Biochem Pharmacol 73:1205-1214. CrossRef Medline

Peyron C, Faraco J, Rogers W, Ripley B, Overeem S, Charnay Y, Nevsimalova S, Aldrich M, Reynolds D, Albin R, Li R, Hungs M, Pedrazzoli M, Padigaru M, Kucherlapati M, Fan J, Maki R, Lammers GJ, Bouras C, Kucherlapati R, et al. (2000) A mutation in a case of early onset narcolepsy and a generalized absence of hypocretin peptides in human narcoleptic brains. Nat Med 6:991997. CrossRef Medline

Peyron C, Tighe DK, van den Pol AN, de Lecea L, Heller HC, Sutcliffe JG, Kilduff TS (1998) Neurons containing hypocretin (orexin) project to multiple neuronal systems. J Neurosci 18:9996-10015. Medline

Prober DA, Rihel J, Onah AA, Sung RJ, Schier AF (2006) Hypocretin/ orexin overexpression induces an insomnia-like phenotype in zebrafish. J Neurosci 26:13400-13410. CrossRef Medline

Ramesh V, Thakkar MM, Strecker RE, Basheer R, McCarley RW (2004) Wakefulness-inducing effects of histamine in the basal forebrain of freely moving rats. Behav Brain Res 152:271-278. CrossRef Medline

Renier C, Faraco JH, Bourgin P, Motley T, Bonaventure P, Rosa F, Mignot E (2007) Genomic and functional conservation of sedativehypnotic targets in the zebrafish. Pharmacogenet Genomics 17: 237-253. CrossRef Medline

Richardson GS, Roehrs TA, Rosenthal L, Koshorek G, Roth T (2002) Tolerance to daytime sedative effects of $\mathrm{H} 1$ antihistamines. $\mathrm{J}$ Clin Psychopharmacol 22:511-515. Medline

Rihel J, Prober DA, Arvanites A, Lam K, Zimmerman S, Jang S, Haggarty SJ, Kokel D, Rubin LL, Peterson RT, Schier AF (2010) Zebrafish behavioral profiling links drugs to biological targets and rest/wake regulation. Science 327:348-351. CrossRef Medline

Roberts F, Calcutt CR (1983) Histamine and the hypothalamus. Neuroscience 9:721-739. Medline

Rossi A, Kontarakis Z, Gerri C, Nolte H, Hölper S, Krüger M, Stainier DY (2015) Genetic compensation induced by deleterious mutations but not gene knockdowns. Nature 524:230-233. CrossRef Medline

Roth T, Roehrs T, Koshorek G, Sicklesteel J, Zorick F (1987) Sedative effects of antihistamines. J Allergy Clin Immunol 80:94-98. Medline

Samaranayake S, Abdalla A, Robke R, Nijhout HF, Reed MC, Best J, Hashemi $P$ (2016) A voltammetric and mathematical analysis of histaminergic modulation of serotonin in the mouse hypothalamus. $\mathrm{J}$ Neurochem 138:374-383. CrossRef Medline

Sander JD, Cade L, Khayter C, Reyon D, Peterson RT, Joung JK, Yeh JR (2011) Targeted gene disruption in somatic zebrafish cells using engineered TALENs. Nat Biotechnol 29:697-698. CrossRef Medline

Schlicker E, Malinowska B, Kathmann M, Göthert M (1994) Modulation of neurotransmitter release via histamine $\mathrm{H} 3$ heteroreceptors. Fundam Clin Pharmacol 8:128-137. Medline

Schöne C, Cao ZF, Apergis-Schoute J, Adamantidis A, Sakurai T, Burdakov D (2012) Optogenetic probing of fast glutamatergic transmission from hypocretin/orexin to histamine neurons in situ. $J$ Neurosci 32:12437-12443. CrossRef Medline
Schwartz JC, Arrang JM, Garbarg M, Pollard H, Ruat M (1991) Histaminergic transmission in the mammalian brain. Physiol Rev 71:1-51. Medline

Seifert R, Strasser A, Schneider EH, Neumann D, Dove S, Buschauer A (2013) Molecular and cellular analysis of human histamine receptor subtypes. Trends Pharmacol Sci 34:33-58. CrossRef Medline

Shan L, Dauvilliers Y, Siegel JM (2015) Interactions of the histamine and hypocretin systems in CNS disorders. Nat Rev Neurol 11:401413. CrossRef Medline

Shigemoto Y, Fujii Y, Shinomiya K, Kamei C (2004) Participation of histaminergic $\mathrm{H} 1$ and noradrenergic alpha 1 receptors in orexin A-induced wakefulness in rats. Brain Res 1023:121-125. CrossRef Medline

Singh C, Oikonomou G, Prober DA (2015) Norepinephrine is required to promote wakefulness and for hypocretin-induced arousal in zebrafish. eLife 4:e07000. CrossRef Medline

Steinman L (2004) Elaborate interactions between the immune and nervous systems. Nature Immunol 5:575-581. CrossRef Medline

Sundvik M, Kudo H, Toivonen P, Rozov S, Chen YC, Panula P (2011) The histaminergic system regulates wakefulness and orexin/hypocretin neuron development via histamine receptor $\mathrm{H} 1$ in zebrafish. FASEB J 25:4338-4347. CrossRef Medline

Sundvik M, Panula P (2015) Interactions of the orexin/hypocretin neurones and the histaminergic system. Acta Physiol (Oxf) 213: 321-333. CrossRef Medline

Thakkar MM (2011) Histamine in the regulation of wakefulness. Sleep Med Rev 15:65-74. CrossRef Medline

Thisse C, Thisse B (2008) High-resolution in situ hybridization to whole-mount zebrafish embryos. Nat Protoc 3:59-69. CrossRef Medline

Torrealba F, Yanagisawa M, Saper CB (2003) Colocalization of orexin a and glutamate immunoreactivity in axon terminals in the tuberomammillary nucleus in rats. Neuroscience 119:1033-1044. Medline

Toyota H, Dugovic C, Koehl M, Laposky AD, Weber C, Ngo K, Wu Y, Lee DH, Yanai K, Sakurai E, Watanabe T, Liu C, Chen J, Barbier AJ, Turek FW, Fung-Leung WP, Lovenberg TW (2002) Behavioral characterization of mice lacking histamine $\mathrm{H}(3)$ receptors. Mol Pharmacol 62:389-397. Medline

Tsatsoulis A (2009) Stress, epigenetics and thyroid autoimmunity. In: The epigenetics of autoimmune disease (Zouali M, ed), pp 135150. Chichester, UK: Wiley-Blackwell.

Valko PO, Gavrilov YV, Yamamoto M, Reddy H, Haybaeck J, Mignot E, Baumann CR, Scammell TE (2013) Increase of histaminergic tuberomammillary neurons in narcolepsy. Ann Neurol 74:794-804. CrossRef Medline

Westerfield M (2000) The zebrafish book. A guide for the laboratory use of zebrafish (Danio rerio). Ed 4. Eugene: University of Oregon Press.

Woods IG, Schoppik D, Shi VJ, Zimmerman S, Coleman HA, Greenwood J, Soucy ER, Schier AF (2014) Neuropeptidergic signaling partitions arousal behaviors in zebrafish. J Neurosci 34:31423160. CrossRef Medline

Yamanaka A, Tsujino N, Funahashi H, Honda K, Guan JL, Wang QP, Tominaga M, Goto K, Shioda S, Sakurai T (2002) Orexins activate histaminergic neurons via the orexin 2 receptor. Biochem Biophys Res Commun 290:1237-1245. CrossRef Medline

Yanai K, Son LZ, Endou M, Sakurai E, Nakagawasai O, Tadano T, Kisara K, Inoue I, Watanabe T, Watanabe T (1998) Behavioural characterization and amounts of brain monoamines and their metabolites in mice lacking histamine $\mathrm{H} 1$ receptors. Neuroscience 87:479-487. Medline

Yokogawa T, Marin W, Faraco J, Pézeron G, Appelbaum L, Zhang J, Rosa F, Mourrain P, Mignot E (2007) Characterization of sleep in zebrafish and insomnia in hypocretin receptor mutants. PLoS Biol 5:e277. CrossRef Medline 